\title{
FPGAs in Industrial Control Applications
}

\author{
Eric Monmasson, Senior Member, IEEE, Lahoucine Idkhajine, Member, IEEE, \\ Marcian N. Cirstea, Senior Member, IEEE, Imene Bahri, Student Member, IEEE, Alin Tisan, Member, IEEE, and \\ Mohamed Wissem Naouar, Member, IEEE
}

\begin{abstract}
The aim of this paper is to review the state of the art of Field Programmable Gate Array (FPGA) technologies and their contribution in industrial control applications. Authors start by addressing various research fields where FPGAs are useful. The features of these devices are then presented followed by their corresponding design tools. To illustrate the benefit of using FPGAs in the case of complex control applications, a sensorless motor controller has been treated. This controller is based on the Extended Kalman Filter. Its development has been made according to a dedicated design methodology which is also discussed. Another example of illustration is the Neural Network Systems. To show the interest of FPGAs in this field, some case studies have been presented.
\end{abstract}

Index terms - Field Programmable Gate Array, Industrial Control Applications, System on Chip, Design Tools, Design methodology, Sensorless Motor Controller, Extended Kalman Filter, Neural Network Systems

\section{I- INTRODUCTION}

Nowadays, to make the difference on the market, new industrial control systems have to be highly performing, very flexible and reliable.

At the same time, the cost is a key issue. In order to reduce it, time-to-market has to be shortened, the price of controller device has to be cheap and its energy consumption as well as the one the controlled system reduced.

This cost reduction is all the more challenging that these new industrial control systems must be based on ever increasing sophisticated control algorithms which need a lot of computing resources and need reduced execution time.

To cope with all these challenges, designers can rely on more and more mature digital electronics technologies that come along now with friendly software development tools.

To implement efficient real-time industrial control systems, designers have the choice between two main families of digital device technologies.

The first family is based on a pure software platform. The associated devices are microcontrollers and Digital Signal Processor controllers (DSP controllers). These components integrate a performing microprocessor core along with several peripherals which are necessary to control the targeted system in real-time and to communicate with the industrial environment.

The difference between microcontrollers [1] and DSP controllers [2] is, for a given surface of silicon, the ratio between the processing unit and the communication and control peripherals. The microcontrollers include a general purpose 16-bit or 32-bit Reduced Instruction Set Computer (RISC) and a wide variety of peripherals, while DSP controllers integrate a high performing processor core based on an hardware accelerator computing block (Multiply and Accumulate Arithmetic Logic Unit MAC ALU) and few peripherals. However, the limits between these two concepts are vanishing since the RISC unit of microcontrollers is more and more powerful and the number and the types of peripherals in DSPs are increasing.

The main advantages of this approach are the maturity of these technologies, the quality of the associated development tools as well as their low price. The main limitations are the difficulty to take advantage of the potential parallelism offered by the control algorithm to be implemented and as a consequence, the limitation of the performances in terms of throughputs and achievable bandwidth.

The alternative family of available digital devices for implementing industrial control systems is the Field Programmable Gate Arrays technology (FPGAs) [3]. These devices consist of pre-designed elementary cells and interconnections that are fully programmable by the end user to build specific hardware architectures that match the requirements of the final targeted application.

The variety of the designed FPGA-based controllers is large. As shown in section II, current FPGAs allow the implementation of efficient 32-bit RISC processors. As a consequence, FPGAs can be first viewed as programmable microcontrollers where designers can combine one or several RISC processors with dedicated peripherals and computing hardware accelerators. From this perspective and due to their ever increasing density, modern FPGAs can be seen as true System-on-Chip (SoC) digital platforms.

To the other side of the range, designers can also design pure specific hardware architectures for stringent applications in terms of performances. Thus, the design and the real-time implementation of control loop with sampling frequency above one $\mathrm{MHz}$ are now possible thanks to this approach as well as the implementation of massive parallel treatments.

Like microcontrollers and DSPs, FPGAs were born in the eighties and are now a mature technology. FPGA design tools are also very friendly. As mentioned before, FPGAs are suited for high speed demanding applications. Indeed, designers can develop a fully hardware architecture which is dedicated to the control algorithm to implement. Hence, by preserving the potential parallelism of the algorithm, the resulting hardware architecture allows matching the expected processing speed specifications.

The main limitation of this technology is the cost. FPGAs are still more expensive compared to their DSP and microcontroller competitors although this claim has to be revised. Indeed, FPGA may be more expensive than a microcontroller but not the cost per implemented function.

Another drawback concerns the difficulty to integrate within current FPGAS mixed Analog/Digital peripherals like $\mathrm{A} / \mathrm{D}$ and $\mathrm{D} / \mathrm{A}$ converters. Once again, this limitation is vanishing with the recent introduction on the market of FPGAs that integrate A/D converters [4].

The success of a recent Special Section devoted to the use of FPGAs to control Industrial Control Systems [5]-[6] 
has demonstrated the great interest of this technology among the research community.

Indeed, the ceaseless increasing density of FPGAs along with their high degree of flexibility pushed designers to use them for controlling a large range of industrial applications. The most significant are now reminded.

A. FPGA-based controllers for embedded industrial and robotic applications

Automotive and aircraft embedded systems are very challenging applications for digital electronics [7]. For these systems, safety is of prime importance. Thus, in [8] and [9] authors have proposed several techniques to tackle this issue and enhance reliability of the FPGA-based controllers.

Another aspect of safety is the possibility for vehicle manufacturers and their suppliers to cope with the obsolescence of critical embedded systems. Electronic devices have now a very limited life time and it is mandatory to still find in the market fully secure FPGAbased Intellectual Property (IP) module to replace them when they are now longer available [10]. The current trend for modular architecture within the FPGA-based embedded controller has also pushed designer to reinforce Intellectual Property by the use of sophisticated encryption techniques [11].

Another concern for automotive and aircraft embedded systems is the design and real-time implementation of high data rate and reliable protocols for in-vehicle networks such as FlexRay or SpW [12]-[13].

Regarding handheld embedded systems, the key issue is the reduction of power consumption [14]-[15]. The notion of power budget is now considered during the design process. In this field, several studies have evolved. In [16], a deep understanding of the source of power consumption and guidelines for its minimization are presented.

FPGA devices are also welcome for managing the communication distributed applications via the efficient real-time Ethernet protocol [17]-[18].

Finally, mention that FPGAs are highly appreciated in the field of robotics. Sensor-based construction of efficient geometric structures via the generalized Voronoi diagram (GVD) have been implemented with success in FPGAs for mobile robots [19-20]. In [21], a coarse-grain parallel deoxyribonucleic acid (PDNA) algorithm for optimal configurations of an omnidirectional mobile robot with a five-link robotic arm is presented.

In [22] and [23] examples are presented of FPGA-based controllers for haptique and tele-operation robotics.

\section{B. FPGA-based controllers for power electronics and drive applications}

A detailed analysis of the benefits of using FPGA-based controllers in power electronics and drive applications can be found in [24]. Just the salient features of this analysis are reminded here. Nowadays, the more limiting factor of current or voltage control loop of a power converter is the limitation of the switching frequency due to switching losses. Thus, the choice of the sampling frequency is most of the time conditioned by the maximum available switching frequency. Based on this analysis, two different groups can be identified:

- High demanding applications

- Constrained switching frequency applications.
This first group concerns applications where the use of FPGA-based controllers is mandatory due to stringent constraints in terms of time or parallelism.

The parallelism constraint case concerns the control of static converters where power is distributed in order to reduce the stress of the power switches. In these cases, concurrency is high since several power channels have to be driven in parallel. A good example of highly parallel operations can be found in [25] where a combined multiphase multilevel (5 phases, 5 levels) Space Vector Pulse Width Modulation (SVPWM) strategy was implemented with success in a low cost FPGA.

The time constraint case concerns applications where the sampling frequency is very high (at least equal or above 100 $\mathrm{kHz}$ ) like for low voltage switch mode power supplies (SMPS). For such applications, the used switching frequency is equal or above $1 \mathrm{MHz}$ FPGA-based controllers are in this case mandatory [26]-[28].

Another high demanding application concerns hardware and power Hardware-In-the-Loop applications (HIL). In [29], authors present a FPGA-based real-time digital simulator of a 3-level 12-pulse Voltage Source Inverter (VSI) fed induction machine drive. It is worth to be mentioned that the VSI model is computed at a fixed timestep of only $12.5 \mathrm{~ns}$, allowing a realistic representation of the IGBT nonlinear switching characteristics and power losses.

The second group consists in applications where sampling is not critical due to switching frequency limitation. For this kind of applications, the use of a software-based controller is possible. However, even in this case, using FPGA can be of great interest since this way, control processing time can be drastically reduced, up to a fraction of the sampling period. This rapidity has an immediate influence on the quality of the control performances, especially when direct control is chosen [30] Thus, the behavior of the proposed digital controller is very closed to its analog counterpart. Besides, due to the ever increasing density of the FPGA components, one can now implement complex algorithms within a few microseconds. A sensorless controller based on the Extended Kalman Filter (EKF) will be discussed later on (section III) to illustrate this trend. Other improvements due to rapidity of the controller are,

- The reduction of complexity: In [31] a simple fault tolerant controller for wind energy conversion systems is presented. It is based on the combination of time and magnitude thresholds which allows reducing significantly the time for default detection (less than $10 \mu \mathrm{s}$ ) where standard solutions need at least one quarter of the fundamental period.

- Synchronization between the current sampler and the PWM carrier. In [32], an FPGA-based implementation of a Switched Reluctance Motor (SRM) current control without anti-aliasing filters is presented. Indeed, by choosing to sample current in the center of a symmetrical modulation, an exact measure of the average current is obtained without any additional filter.

- Shared control resources: It consists in controlling several similar plants with a unique controller. Thus, in [33], authors have proposed an FPGA-based controller that is able to control up to 4 AC-drives with a sampling period of only $50 \mu$ s. Other interesting multi-axis controllers are given in [34]. 
- Oversampling strategies which are mainly used for improving the quality of the measurement of quantities. For instance, in [35], authors present a quasi-ideal direct measuring of the mean value of the current feedback.

- Adding of new control functionalities: From this perspective, a real-time processing extension that naturally comes to mind is the implementation of a predictive control strategy like the one presented in [34] for synchronous motor drives. Another exciting challenge is the addition of health-monitoring processing. Very promising works have already been proposed for SMPS applications, like in [36] where an on-line identification strategy through crosscorrelation was successfully implemented. In the same vein, diagnosis algorithms can also be implemented. To this purpose, [37] and [38] have proposed wavelet-based diagnosis algorithms for respectively induction motor broken-rotor-bar detection and fault detection and classification in transmission lines.

This paper is aimed to explain the importance of FPGAbased solutions in the design of industrial control systems. The second section recalls the main features of the available FPGA technologies. Then, in the third section, an in-depth of a complex FPGA-based controller example is treated. It consists in a FPGA-based sensorless controller for synchronous motor using an Extended Kalman Filter (EKF). All the steps of the design are described and comparisons are given in terms on software and hardware implementations. Section IV is devoted to Artificial Neural Network (ANN) based FPGA-Systems. There is a growing interest for implementing ANN within FPGA mostly because their inherent parallelism. Two case studies are presented: a highly compact PWM generator and an electronic nose. Finally, conclusions are given and future trends given.

\section{II- PRESENTATION OF FPGAS}

Since their first introduction to the market in 1985 by the Xilinx Company, FPGA hardware technologies have attracted an always increasing interest and have significantly disrupted the early digital development process trends. Indeed, these devices belong to the so called semi-custom ASICs (Application Specific Integer Circuits). The latter low cost devices consist of pre-designed (by the manufacturer) elementary cells and interconnections that can be programmed and interconnected by the user. This has the credit to allow rapid-prototyping solutions and make the design process more flexible and cheaper. This is not the case of full-custom ASICs that are manufactured for a specific application and cannot be re-programmed by the user.

A. Generic structure of an FPGA.

As presented in Fig. 1, the basic structure of an FPGA consists of a sea of Logic Blocks (LBs), of an interconnection network and configurable $\mathrm{I} / \mathrm{O}$ blocks. Because of their very high level of integration, in the recent FPGA devices are also included memory blocks, hardwired DSP blocks, clock manager blocks and communication blocks [41].

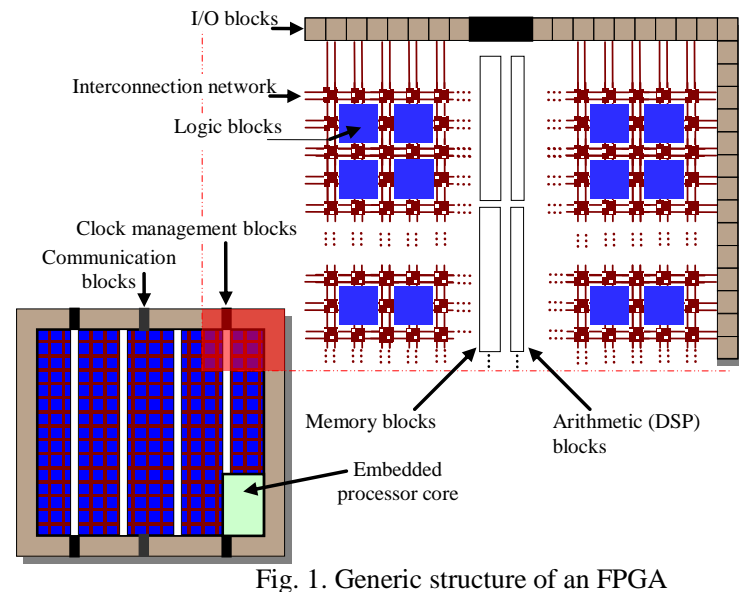

Depending on the expected function to implement, each LB is configured to perform combinatorial and/or sequential operations. For the combinatorial operations, a set of LookUp-Tables (LUTs) are included. This is the same for the sequential operations with a set of D-Flip-Flops. An LB is also able to perform a local storage function (distributed RAM memory), shift register (SR), multiplexer, and adder/subtractor operations. The interconnection network is also programmable by the user so as to interconnect as many LBs as necessary.

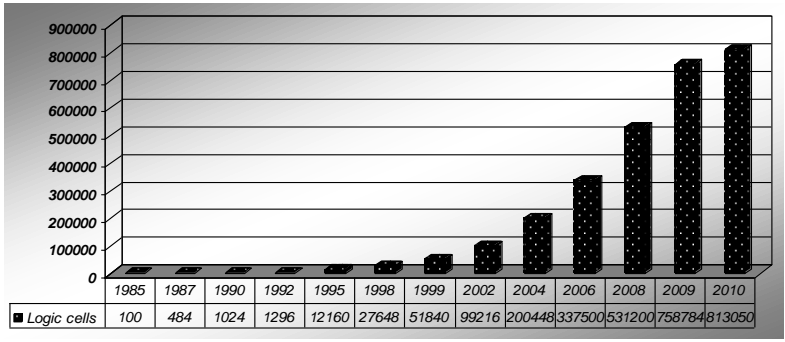

Fig. 2. Evolution of the FPGA density

On the other hand, the internal structure of LBs differs from an FPGA family to another. For this reason, a normalized FPGA density metric has been accepted. Indeed, a common Logic Cell (LC) has been defined. It consists of a 4-bit LUT, a D-Flip-Flop, a carry chain (for arithmetic operations) and a multiplexer, [39]-[42]. Fig. 2 presents the evolution of FPGAs in terms of density (i.e. number of the available LCs) since 1985. The waveform has been obtained after a comparison of the commercialized FPGA devices within each year.

The configurable I/O blocks allow the interfacing between the internal architecture and the external environment.

In order to optimize FPGA resources, hardwired DSP blocks (arithmetic blocks) including multipliers, adders and accumulators are included. For the same purpose, memory blocks (RAM, ROM, Flash RAM) are also integrated.

The integrated clock manager blocks allow the management of the clocking resources. They are commonly based on Phase-Locked-Lools (PLLs). The latter support several features such as frequency multiplication and division, propagation delay compensation and phase shift correction.

The current FPGA devices include also communication blocks that consist generally of transmission and reception buffers. Various communication protocols are supported, including among others USB, Ethernet, CAN, PCI, SPI and I2C protocols. 
To provide high integration density, high speed and low power consumption, FPGAs have been the subject of a considerable progress in terms of copper process technology. Recent devices are now reaching down to 40 $\mathrm{nm}$ copper process $(28 \mathrm{~nm}$ has been recently announced by Xilinx and Altera vendors) [39], [40]. Fig. 3 shows the evolution of the process technology since 1985.

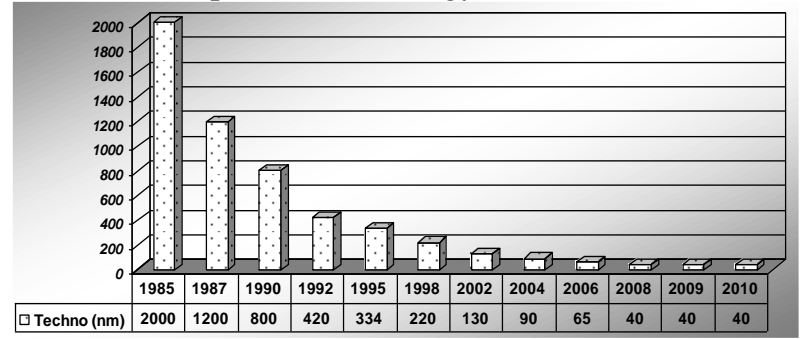

Fig. 3. Evolution of the FPGA process copper technology

The internal structure, the operating mode and the configuration of these FPGA elements differ depending on the device family and technology. There are various configuration technologies including, SRAM, EPROM, EEPROM, Fuse, Antifuse and Flash technologies. Because of their popularity, only the SRAM and the Flash technologies will be discussed.

Besides the evolution of these FPGA technologies and in order to meet flexibility and high integration capability, the novel FPGA devices give the possibility to implement an increasing diversity of cores. Indeed, in addition to the previously presented FPGA cores and as it will be discussed afterwards, FPGA can integrate one or several processors and even analog peripherals [45]-[53]. They are then considered as System-On-Chips (SoCs) or System-onProgrammable-Chips (SoPCs) solutions.

\section{B. FPGA internal technologies and architectures}

\section{1- SRAM based FPGAs}

The configuration of an SRAM-based FPGA is entirely made using a set of dedicated SRAM blocks. These blocks are organized as a specific configuration layer. This programming strategy is volatile and a reconfiguration of the devices is required after the device is switched off. The most popular SRAM-based FPGA families are Xilinx and Altera families, [39], [40]. Among the commercialized FPGA devices, one can stress the high performance VIRTEX (Xilinx) and STRATIX (Altera) FPGAs and the low cost SPARTAN (Xilinx) and CYCLONE (Altera) FPGAs. In the field of industrial control applications, cost is a key-issue. As a consequence, the low cost families are preferred. Thus, only the latest Xilinx SPARTAN-6 and Altera Cyclone-4 FPGA families will be presented.

Xilinx SPARTAN-6 FPGA: As shown in Fig. 4, this SRAM-based FPGA incorporates a sea of CLBs (Configurable Logic Blocks) and an interconnection network [39]. Roughly speaking each CLB is equivalent to 12.8 LCs.

A CLB contains a pair of slices, the SLICEX and SLICEL/SLICEM. Each slice can be configured to perform combinatorial functions using four 6-bit LUTs and sequential functions using eight D Flip-Flops. The SLICEM can also be configured to perform a distributed RAM block for data storage [39]. Consequently, there are $50 \%$ of SLICEX, $25 \%$ of SLICEM and $25 \%$ of SLICEL inside the SPARTAN-6 device (see Fig. 4).

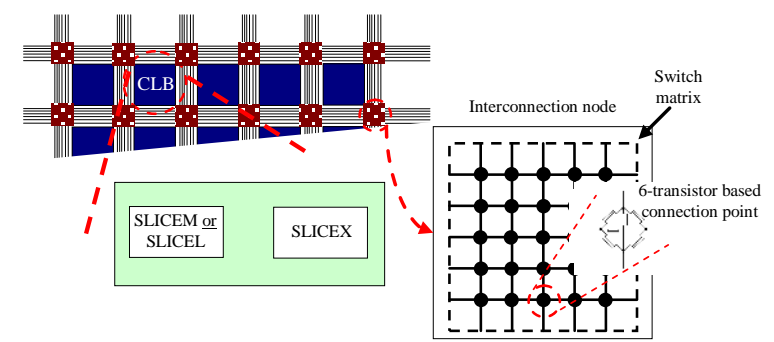

Fig. 4. SRAM-based SPARTAN-6 FPGA

Altera CYCLONE-4 FPGA: The structure of the Cyclone-4 FPGA is presented in Fig. 5. In this case, the logic cells are named Logic Elements (LE). Roughly speaking each LE of an Altera FPGA is equivalent to one LC.

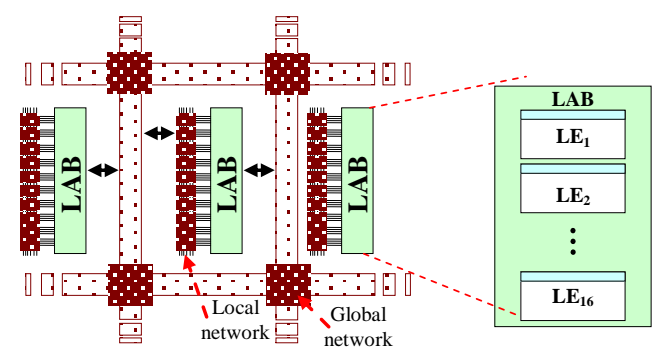

Fig. 5. SRAM-based CYCLONE-4 FPGA

The LEs are gathered in 16-group blocks called Logic Array Blocks (LAB). A LE consists of a 4-bit LUT that can perform either combinatorial or arithmetic operations and a D-Flip-Flop for sequential operations. The interconnection blocks are organized in 2 levels; local interconnection network and global network, [40].

Table I: SRAM-based FPGA - some specifications

\begin{tabular}{|c|c|c|}
\hline & SPARTAN 6 & CYCLONE 4 \\
\hline Number of LCs & 147443 (11519 CLBs) & 150000 \\
\hline $\mathrm{I} / \mathrm{Os}$ & 576 & 532 \\
\hline DSP blocks & 180 DSP blocks & 360 Multipliers \\
\hline $\begin{array}{l}\text { Clocking performances: } \\
\text { PLL output frequency range }\end{array}$ & 400-1080 MHz & 600-1300 MHz \\
\hline Memory size (RAM, ROM) & $\begin{array}{c}6179 \text { Kb (RAM blocks } \\
\text { and distributed RAM) }\end{array}$ & $\begin{array}{c}\mathbf{6 4 8 0} \text { Kb (RAM } \\
\text { blocks) }\end{array}$ \\
\hline Process technology & $45 \mathrm{~nm}$ & $60 \mathrm{~nm}$ \\
\hline
\end{tabular}

Table I presents some important specifications regarding the SRAM-based FPGAs (SPARTAN-6 and CYCLONE-4). It indicates the maximum available characteristics depending on the FPGA series.

\section{2- Flash based FPGA}

In the case of Flash technology, the configuration is based on flash connections that keep the configuration state when the power is off. Each connection contains two transistors that share a floating gate and stores the programming information, [4]. This technology is the most useful technology in aircraft and space systems since it guaranties the configuration against the SEU (Single Event Upset) radiations. In the proposed survey, the Actel Fusion Flash-based FPGA is presented. Its internal logic cells consist of VersaTiles (VTs). The latter can implement either a combinatorial (3-bit LUT) or a sequential (D Flip Flop) functions. Fig. 6 gives an overview of the Fusion FPGA structure. 


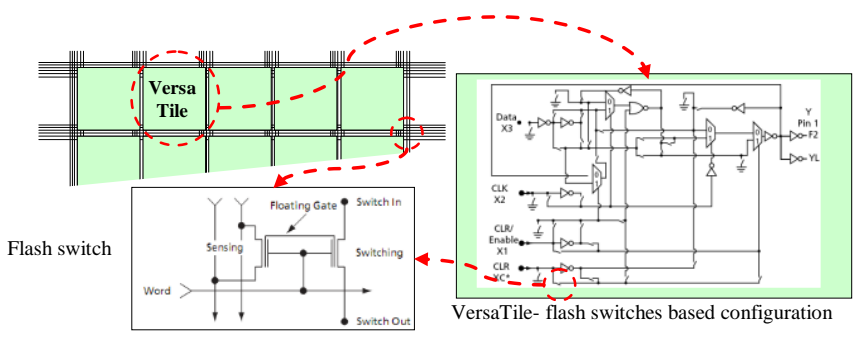

Fig. 6. Flash-based Fusion FPGA

Table II highlights some of the internal resources and performances of the described Flash-based Fusion FPGA.

Table II: Flash-based Fusion FPGA- some specifications

\begin{tabular}{|c|c|}
\hline & Fusion \\
\hline Number of VersaTiles & Up to $38.5 \mathrm{~K}$ \\
\hline Number of I/Os & Up to 252 \\
\hline DSP blocks (arithmetic blocks) & $\mathbf{0}$ \\
\hline $\begin{array}{l}\text { Clocking performances: } \\
\text { PLL output frequency range }\end{array}$ & $350 \mathrm{MHz}$ \\
\hline $\begin{array}{l}\text { Memory size (RAM/FIFO, } \\
\text { ROM, Flash) }\end{array}$ & $\begin{array}{l}270 \mathrm{~Kb} \text { (RAM); } 1 \mathrm{~Kb} \\
(\mathrm{ROM}) ; 8 \mathrm{Mb} \text { (Flash) }\end{array}$ \\
\hline Process technology & $130 \mathrm{~nm}$ \\
\hline
\end{tabular}

C. FPGA System on Chip (SoC) trends

\section{1- Processor unit}

As mentioned in the introduction, SoC approaches present new design features allowing the combination of software and hardware designs. Two categories of processor cores are considered, the "non-synthesizable" cores and the "synthesizable" cores.

The non-synthesizable (also called hard processor cores) have a custom VLSI layout (in dedicated silicon) that is integrated within the FPGA. As a general rule, a hard processor core offers higher clock speeds with less flexibility. For example, Altera provides an ARM9 processor core embedded in its EPXA10 series that is marketed as an Excalibur ${ }^{\mathrm{TM}}$ device [40]. The Xilinx Virtex5 integrates also a hardwired PowerPC 440 processor cores on-chip [39]. Recently, Actel has proposed the first hardwired Cortex-M3 processor core integrated into its Fusion FPGA family [4].

The synthesizable (Soft cores), such as Altera's Nios II, Xilinx's MicroBlaze processors and Actel's ARM7 or Cortex-M1, use existing FPGA logic cells to implement the processor core. The particularity of such approach is the flexibility that allows the designer to configure and specify the number, the types of peripherals, the memory width... However, these cores have slower clock rates.

\section{2- Analog peripherals}

In addition to the processor unit, Actel's Fusion family offers a new level of integration by allowing designer to implement a mixed signal treatment (analog and digital). It integrates a 12-bit programmable Successive Approximation Register ADC (SAR-ADC). The SmartFusion device integrates two ADCs. These features make this FPGA SoC device suitable for control applications [44].

\section{3-Multiprocessing architectures in a single chip}

In case of complex digital applications, very high demanding performances can be achieved via the use of heterogeneous multiprocessing architectures in a single chip (MPSoCs). Indeed, such alternative provides high level of scalability compared to monolithic cores, in particular in terms of power and performance [45]-[48]. Important issues in MPSoC design are the communication infrastructure and task mapping. In fact, most of these complex designs are based on dedicated channels or shared buses due to their low cost.

Unfortunately, scalability is restricted by serialization for multiple bus access requests. A promising approach for this issue is the Networks on Chip (NoCs) concept. The latter provides reusability, well-controlled global wire delay and efficient global communication [48]-[52]. In this context, the use of a system language like SystemC can be of interest. This allows simulating the complete multiprocessor system and the communication strategy at the cycle-accurate and signal-accurate level.

Table III: Features of processor cores for SoCs [39], [40], [4], [63]

\begin{tabular}{|c|c|c|c|c|c|}
\hline \multicolumn{2}{|c|}{$\begin{array}{c}\text { Features } \\
\text { SoC }\end{array}$} & $\underset{\text { Virtex-5 }}{\text { Xilinx }}$ & $\begin{array}{c}\text { Actel } \\
\text { Fusion } 1\end{array}$ & $\begin{array}{c}\text { Altera } \\
\text { Startix III }\end{array}$ & $\begin{array}{c}\text { Texas } \\
\text { Intruments1 }\end{array}$ \\
\hline \multicolumn{2}{|l|}{$\mathrm{CPU}$} & $\begin{array}{c}\text { PowerPC } \\
440\end{array}$ & Cortex-M1 & $\mathrm{NIOS} \mathrm{II/f}$ & DSP C 28x \\
\hline \multicolumn{2}{|c|}{ Frequency $(\mathrm{MHz})$} & 400 & 60 & 290 & 150 \\
\hline \multicolumn{2}{|c|}{ Bit Number } & $32 \mathrm{~b}$ & $16 / 32 b$ & $32 \mathrm{~b}$ & $32 \mathrm{~b}$ \\
\hline \multicolumn{2}{|c|}{ Pipeline stages } & $\begin{array}{c}\text { RISC } \\
\text { superscalar } \\
\text { 7-stage } \\
\text { pipeline }\end{array}$ & $\begin{array}{l}\text { RISC, 3- } \\
\text { stage } \\
\text { pipeline }\end{array}$ & $\begin{array}{c}\text { RISC, 6- } \\
\text { stage } \\
\text { pipeline }\end{array}$ & $\begin{array}{c}\text { DSP, 8- } \\
\text { stage } \\
\text { pipeline }\end{array}$ \\
\hline \multicolumn{2}{|c|}{ Multiplier } & $\begin{array}{c}\text { Hardware } \\
32 \times 32 \\
\text { multiplier } \\
\text { (single cycle) }\end{array}$ & $\begin{array}{l}\text { Synthesizabl } \\
\text { e } 32 \times 32 \\
\text { multiplier } \\
\text { (3 cycle for } \\
\text { standard } \\
\text { multiplier and } 33 \\
\text { cycle for small } \\
\text { multiplier) }\end{array}$ & $\begin{array}{c}\text { Hardware } \\
32 \times 32 \\
\text { multiplier } \\
\text { (single cycle) }\end{array}$ & $\begin{array}{c}\text { Hardware } \\
32 \times 32 \\
\text { multiplier } \\
\text { (single } \\
\text { cycle) }\end{array}$ \\
\hline \multirow[t]{3}{*}{$\mathrm{ADC}$} & Mode & & SAR -12b & & $\begin{array}{c}\text { Pipeline- } \\
12 \mathrm{~b}\end{array}$ \\
\hline & Rate & & $600 \mathrm{Ksps}$ & & $12.5 \mathrm{Msps}$ \\
\hline & pipeline & & 30 & & 16 \\
\hline \multicolumn{2}{|c|}{ Logic cells Usage } & & 4353 & 1020 & \\
\hline \multicolumn{2}{|c|}{ Synthesizable } & No & Yes & Yes & No \\
\hline \multicolumn{2}{|c|}{ Configurability } & ++ & ++ & ++ & -- \\
\hline \multicolumn{2}{|c|}{ Performance } & ++ & + & + & ++ \\
\hline
\end{tabular}

Legend: ++ good; + Moderate; -- Poor

For these applications, the embedded Real Time Operating System (RTOS) becomes essential. In fact, RTOS offers a rich set of services which provides a basic support to the application and represents in a great part the way in which software is safely reused in an embedded system. Examples of used RTOS are Embedded Linux, MicroC/OSII... [53].

Table III presents a comparison between several SoC solutions: a standard DSP controller device, a PowerPC hard core, a Cortex-M1 and NIOS II/f soft core.

\section{Design tools}

In parallel to the FPGA features evolution, the design tools have become mature as well. Today, FPGA vendors provide a fairly complete set of tools that allow high quality design process starting from the hardware description, using VHDL or Verilog languages, to the final bitstream generation [39]-[42]. A simplified synoptic of the FPGA design process is presented in Fig. 7.

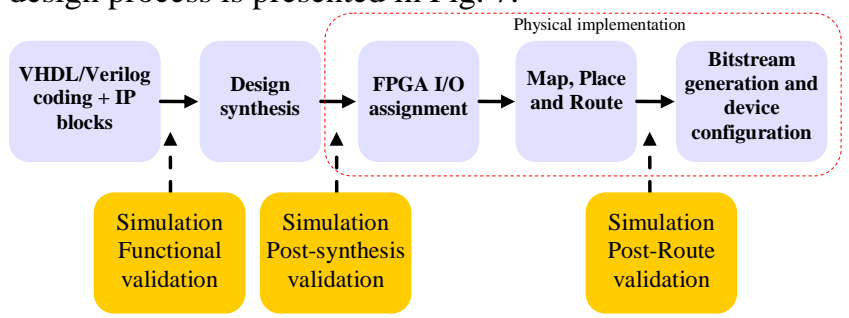

Fig. 7. Simplified synoptic of the FPGA design process 
Generally, design tools include hardware design and verification tools (VHDL/Verilog editor, synthesizer, place/route and physical implementation tools), vendor libraries in addition to simulation and debugging tools. Some examples are the Integrated Software Environment (ISE) tools from Xilinx, Quartus tools from Altera and Libero Integrated Design Environment (LiberoIDE) tools from Actel. All of them provide flexible and complete design features with additional associated tools for simulations (e.g. ModelSim tools) and for debugging (e.g. ChipScope tools from Xilinx).

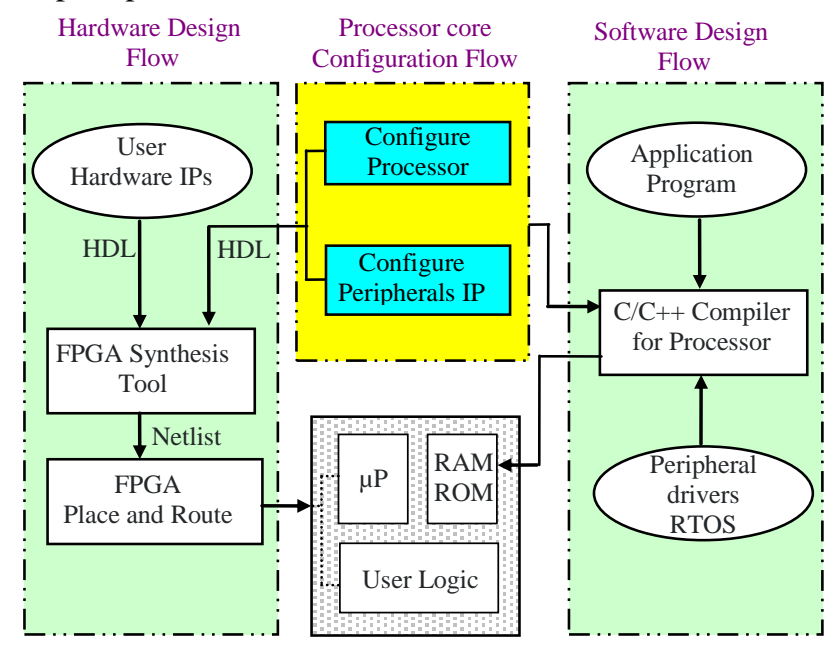

Fig. 8. Typical FPGA SoC design flow

Furthermore, to suit SoC trends, FPGA vendors provide software development tools (editor, compiler, assembler, linker and debugger), software vendor IPs and processor customization tools. For example, Xilinx provides Embedded Development Kit (EDK) platform, Altera provides Embedded Design Suite (EDS) platform and Actel provides SoftConsole platform. Fig. 8 presents a standard design flow for developing SoC applications.

This design flow consists of two main procedures: the software design Flow and the hardware design Flow. It offers a user-friendly interface that allows the designer to customize the processor for a specific project. After its configuration, the processor core is generated in the form of an HDL file (in the case of Altera and Actel tools) or a netlist file (in the case of Xilinx tools). Then, this file can be associated to custom user logic and integrated within the hardware design flow to be synthesized, placed and routed. The FPGA can be configured with the resulting bistream file. Then, the program which will be integrated on the soft processor cores can be compiled with the associated library files and $C$ header files. A $\mathrm{C} / \mathrm{C}++$ compiler targeted for this processor is also provided for the development system.

III- FPGA-BASED DESIGN OF AN INDUSTRIAL CONTROLLER CASE STUDY: SENSORLESS MOTOR CONTROLLER BASED ON AN EXTENDED KALMAN FILTER

FPGA technology allows the development of hardware architectures within a flexible programmable environment. This feature gives designer an additional degree of freedom compared to software implementations based on microcontrollers and DSPs [42], [43]. This is because FPGAs are outperforming these software solutions by exploiting the inherent parallelism of the algorithm. Consequently, designer can develop a hardware architecture that is fully dedicated to the algorithm to implement. Thus, the execution time is drastically reduced. Regarding an industrial control application, the reduction of the execution time makes the control quasi-instantaneous. The control bandwidth is then enhanced.

On the other hand, the development of a dedicated hardware architecture can be seen as intuitive and not adapted to the implementation of more and more complex controllers. This is the reason why the use of a wellstructured design methodology is quite important. Such a methodology should consist of a set of steps and rules to be followed in order to make the design process more manageable and less intuitive.

In this section, the FPGA-based design of an industrial controller is discussed. In order to show the benefit of using FPGA for complex controllers, a sensorless motor controller is presented. This controller uses an Extended Kalman Filter (EKF) to estimate the rotor speed and position of the used synchronous motor. This development is made according to the design methodology, [42]-[44], overviewed in Fig. 9.

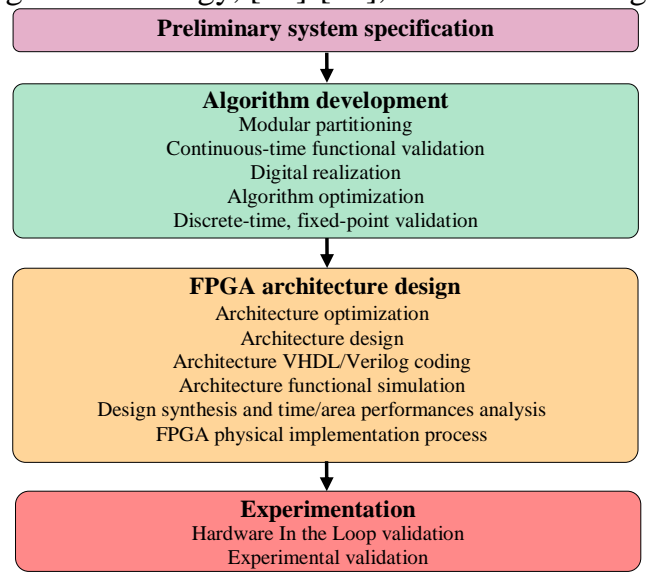

Fig. 9. FPGA-based controller design methodology

The particularity of this methodology consists in providing a top-down design process that starts from the preliminary system specification to the final experimental validation. In addition, a notable distinction between the development of the algorithm and its digital implementation is made. This distinction has the credit of making the algorithm totally independent of the used digital device. For instance, once the developed algorithm is achieved, either a hardware solution (FPGA) or a software solution (DSP) can be chosen. Furthermore, this distinction can lead to a separation between the needed designer qualifications. For example, the algorithm development may be realized by control engineers and the FPGA development by a microelectronics expert.

From a more technical point of view, the proposed design methodology includes optimization assumptions that ought to be achieved so as to adapt the algorithm complexity to the available FPGA resources. As it will be discussed later, this optimization is done during the algorithm development process and during the FPGA architecture development one. For the first case, this consists in reducing the computational cost of the algorithm (reduction of the number of processed operations). As for the second case, this consists in studying the data dependency of the algorithm and finding out the potential factorizations that lead to the use of a minimum of operators that process a maximum of operations. This optimization can be achieved by applying for example the so-called Algorithm Architecture Adequation $\left(\mathrm{A}^{3}\right)$ methodology [30].

A. Preliminary system specification

In this first step, the preliminary specification of the whole sensorless control application is made. To this aim, a 
hardware specification and an algorithm benchmarking are achieved.

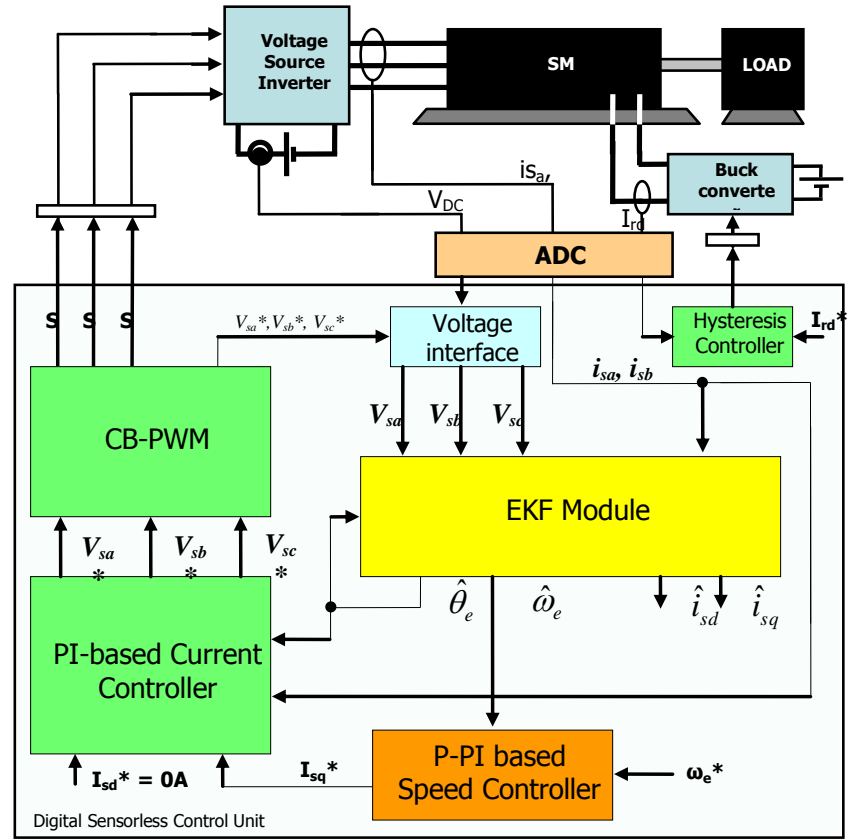

Fig. 10. Sensorless control system

The hardware specification consists in choosing, depending on the load conditions, the AC motor to be controlled and the appropriate power supply system. In addition, the sensors, the digital control unit and the ADC interfaces are defined. The algorithm benchmarking consists in choosing the control strategy and in specifying the sensorless method.

In the proposed application, the controlled AC drive consists of a salient Synchronous Motor (SM) fed by a Voltage Source Inverter (VSI). The sensorless controller is based on an Extended Kalman Filter (EKF) which estimates the rotor position and speed [54]-[62]. An overview of the implemented control system is presented in Fig. 10.

The EKF estimation is based on the normalized $d-q$ stochastic system model (relations 1-3). This model is derived under the "infinite inertia" hypothesis [54]-[62].

$$
\begin{aligned}
& \dot{x}_{n}=f\left(x_{n}, u_{n}\right)+w \\
& y_{n}=H \cdot x_{n}+v \\
& x_{n}=\left[\begin{array}{llll}
\frac{i_{s d}}{I_{B}} & \frac{i_{s q}}{I_{B}} & \frac{\omega_{e}}{\omega_{B}} & \frac{\theta_{e}}{\theta_{B}}
\end{array}\right]^{T} ; u_{n}=\left[\begin{array}{ll}
\frac{v_{s d}}{V_{B}} & \frac{v_{s q}}{V_{B}}
\end{array}\right]^{T} ; y_{n}=\left[\begin{array}{ll}
\frac{i_{s d}}{I_{B}} & \frac{i_{s q}}{I_{B}}
\end{array}\right]^{T}
\end{aligned}
$$

$$
f\left(x_{n}, u_{n}\right)=\left[\begin{array}{c}
\frac{-R_{s}}{L_{s d}} \cdot i_{s n d}+\frac{L_{s q}}{L_{s d}} \cdot \omega_{B} \cdot \omega_{e n} \cdot i_{s n q} \\
\frac{-R_{s}}{L_{s q}} \cdot i_{s n q}-\left(\frac{L_{s d}}{L_{s q}} \cdot i_{s n d}+\frac{M_{s r} \cdot I_{m d}}{L_{s q}}\right) \cdot \omega_{B} \cdot \omega_{e n} \\
0 \\
\frac{\omega_{B}}{\theta_{B}} \cdot \omega_{e n}
\end{array}\right]+\left[\begin{array}{cc}
\frac{V_{B}}{I_{B} \cdot L_{s d}} & 0 \\
0 & \frac{V_{B}}{I_{B} \cdot L_{s q}} \\
0 & 0 \\
0 & 0
\end{array}\right] \cdot u_{n} ; \quad H=\left[\begin{array}{cc}
1 & 0 \\
0 & 1 \\
0 & 0 \\
0 & 0
\end{array}\right]^{T}
$$

Where $f$ and $H$ are the state space and the output matrices. $x_{n}$ is the normalized state space vector. $u_{n}$ and $y_{n}$ are respectively the normalized system input and output vectors. $\left(i_{s d}, i_{s q}\right)$ and $\left(v_{s d}, v_{s q}\right)$ are respectively the $\mathrm{d}-\mathrm{q}$ stator currents and stator voltages. $\left(L_{s d}, L_{s q}\right)$ are the stator inductances and $R_{s}$ is the stator resistance. $\theta_{e}$ and $\omega_{e}$ are respectively the rotor position and speed. $V_{B}, I_{B}, \omega_{B}, \theta_{B}$ are the base values for normalisation. $n$ is the normalization index. The model and measurement disturbances are statistically described by the zero-mean Gaussian noises $w$ and $v$ respectively characterized by covariance matrices $Q$ and $R$.
In the following, we are going to present the EKF basics. To start with, relation (4) presents the discrete-time stochastic state space model of the observed system. $f_{d}$ is the discrete time state space matrix and $k$ is the sampling index. Table IV summarizes the EKF equations.

\begin{tabular}{|c|c|c|}
\hline \multicolumn{3}{|l|}{ Prediction Step } \\
\hline \multicolumn{2}{|c|}{$\hat{X}_{n k / k-1}=f_{d}\left(\hat{X}_{n k-1 / k-1}, u_{n k-1}\right)$} & (5) \\
\hline \multicolumn{3}{|l|}{ EKF Compensator } \\
\hline Jacobian matrix : & $F_{d n k}=\left.\frac{\partial\left(f_{d}\right)}{\partial x}\right|_{x=\hat{x}_{n-j}}$ & (6) \\
\hline $\begin{array}{l}\text { Covariance matrix } \\
\text { prediction : }\end{array}$ & $\begin{array}{r}P_{n k / k-1}=F d_{n k} \cdot P_{n k-1 / k-1} \cdot F d_{n k}^{t}+Q \\
\text { Initial condition } \mathrm{P}_{0}\end{array}$ & (7) \\
\hline $\begin{array}{l}\text { Kalman gain } \\
\text { calculation : }\end{array}$ & $K_{n k}=P_{n k / k-1} \cdot H^{t} \cdot\left(H P_{n k / k-1} H^{t}+R\right)^{-1}$ & (8) \\
\hline $\begin{array}{c}\text { Updating } \\
\text { covariance matrix : }\end{array}$ & $P_{n k / k}=P_{n k / k-1}-K_{n k} H P_{n k / k-1}$ & (9) \\
\hline \multicolumn{3}{|l|}{ Innovation step } \\
\hline$\hat{X}_{n k / k}=\hat{X}_{n k /}$ & & (10) \\
\hline
\end{tabular}

$$
\begin{aligned}
& x_{n k}=f_{d}\left(x_{n k-1}, u_{n k-1}\right)+w_{k} \\
& y_{n k}=H \cdot x_{n k}+v_{k}
\end{aligned}
$$

Table IV: EKF Algorithm

The implemented stator current controller is based on Anti-windup PI regulators [30], [53]. This PI controller calculates the $\mathrm{d}-\mathrm{q}$ voltage references according to the measured and reference currents. After a coordinate transformation, the 3-phase voltage references are processed. Then the used Carrier-Based Pulse Width Modulation (CB-PWM) generates the PWM signals for the VSI. The speed controller is made up using a ProportionalProportional Integral (P-PI) regulator that is deeply studied in [53].

As far as rotor current is concerned, a hysteresis controller and a buck converter have been implemented in order to maintain the rotor current equal to a constant value [30]. The voltage interface aims to generate the 3-phase stator voltages after a multiplication of the per-unit voltages (from the controller) by the measured DC link voltage.

\section{B. Algorithm development}

The algorithm development process consists of a set of steps during which the designer makes the functional validation and prepares the algorithm for digital implementation.

\section{1- Modular partitioning}

The modular partitioning consists in dividing the whole sensorless algorithm into independent and reusable modules with different levels of granularity. For the chosen example, the extracted modules are those presented in Fig. 10.

\section{2- Continuous-time functional validation}

Once the control system is designed and the algorithm partitioning is made, a continuous-time (s-domain) functional simulation is achieved using Matlab/Simulink tools. This step is aimed to simulate and verify the functionality of the complete control system.

\section{3- Digital realization}

During this step, the first task consists in making a digital synthesis of the aimed control closed loop. Two approaches are considered, the direct synthesis approach and the digital re-design approach. The first one consists in configuring the controller and synthesizing the used regulators in a fully discrete-time z-domain. The re-design approach consists in synthesizing regulators in the 
continuous s-domain and then making the convenient transformation to the discrete-time domain ( $\mathrm{ZOH}$, Tustin, Euler).

The speed and current regulators have been synthesized using the re-design approach using the Tustin transformation. The EKF has been synthesized using the direct synthesis approach but its prediction module is based on the re-designed system model. For the latter, the Euler transformation method is used. The chosen sampling frequency is set to $10 \mathrm{KHz}$.

The obtained digital controller and observer can be considered as digital filters that are now to be realized. Their structures are then specified (direct form, cascade form, transpose form ...).

The following task is the choice of the fixed point data format. This choice can be made in two stages [64]. The first one is the choice of the fixed-point format of the coefficients by studying the stability of the closed-loop. The second stage concerns the choice of the fixed-point format for the variables. To this purpose, the limit-cycle at steady state and the signal-to-noise ratio are both considered.

A simpler but more intuitive method for choosing the fixed-point format is by trial-and-error fixed-point simulations. Indeed, designer can develop the fixed-point model and then make a comparison with the floating point initial model. The format that leads to a minimum quantification error is then maintained. Another and still more intuitive way to choose the format is the use of Matlab/Simulink fixed-point tool. At the end of each simulation, this tool collects information about the processed data and displays their maximum, minimum values. It also indicates when overflows occur. Then, these data ranges help designer to choose the appropriate fixed point format.

In the proposed application, the $s[22 Q 20]$ fixed-point format is used for the EKF estimation, the $s$ [17Q16] format for the speed controller and the $s[13 Q 12]$ format for the current controller. This representation is labeled as $s[(i+f) / Q f]$ for signed data. $(i+f)$ is the total data size, $i$ is the number of bits of the integer part and $f$ is the number of bits of the fractional part.

\section{4- Algorithm optimization}

As mentioned before, an optimization is to be performed in order to reduce the number of operations. This optimization is quite mandatory in the case of the FPGA solution since the size of the developed architecture is conditioned with the complexity of the algorithm. For instance, a complex control algorithm, where many greedy operations like multiplications have to be processed, needs a rigorous and smart simplification without losing the required performances.

In order to illustrate the importance of optimizing the complexity of the developed algorithm, the matrix-based Kalman compensator is focused on (Table IV). Assuming that $P_{k / k-l}$ and $P_{k / k}$ are symmetrical and $Q$ and $R$ are diagonal, such matrix treatment can be replaced advantageously by scalar treatment with a significant reduction of the number of operations and processed variables [62]. This is clearly demonstrated by the table $\mathrm{V}$, where the complexity in terms of arithmetic operations is evaluated.

\begin{tabular}{|c|c|c|}
\hline \multicolumn{2}{|c|}{ Table V: complexity of the Kalman compensator } \\
\hline & $\begin{array}{c}\text { Initial Kalman } \\
\text { compensator }\end{array}$ & $\begin{array}{c}\text { Optimized Kalman } \\
\text { compensator }\end{array}$ \\
\hline Multiplications & 318 & 149 \\
\hline Additions & 244 & 107 \\
\hline
\end{tabular}

\begin{tabular}{|c|c|c|}
\hline Subtractions & 16 & 11 \\
\hline Inversions & 1 & 1 \\
\hline
\end{tabular}

However, depending on the available hardware resources, the obtained complexity could remain inadequate to the FPGA implementation. As a result, an optimization of the FPGA-based architecture is also required. This point will be discussed during the FPGA-based architecture design.

\section{5-Discrete-time and fixed-point simulation}

After having developed the aimed digital control algorithm and having specified the suitable sampling frequency and the FWL, designer has to make a final functional verification by simulating the developed algorithm in the discrete-time and fixed-point domain with the help of Matlab/Simulink tools. In the case of the chosen example, the obtained simulation results are shown in Fig. 11 where the waveforms of the measured and estimated rotor speed and position are given.

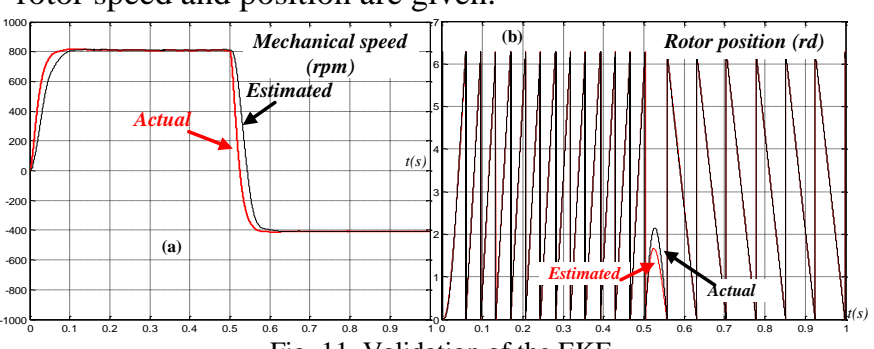

Fig. 11. Validation of the EKF

Estimation of the speed (a) and position (b)

\section{FPGA-based architecture design}

In the case of having chosen the FPGA target to implement the developed algorithm, designer initiates the development of the corresponding FPGA-based architecture. To make the design process less constraining, an interesting solution consists in generating automatically the FPGAbased architecture from Matlab/Simulink, using dedicated toolboxes proposed by FPGA manufacturers [65]. However, in case of complex algorithms, this solution can lead to unoptimized architecture that may be inadequate to the available FPGA resources. This is the reason why, in the proposed design methodology, designer has to develop and code himself the FPGA-based architecture with the help of the following steps (Fig. 9).

\section{1 - Architecture optimization $-A^{3}$ methodology}

The optimization of the FPGA-based architecture is based on the $\mathrm{A}^{3}$ methodology. Presented in [30], the latter consists in studying the parallelism of the algorithm so as to find the potential factorization. The aim is to use a minimum of operators that process a maximum of operations. Two graphical representations are introduced: the Data Flow Graph (DFG) and the Factorized DFG (FDFG). This last includes specific nodes called, F ("Fork"), J ("Join"), D ("Diffuse") and I ("Iterate"). These nodes are used to delimit the factorization borders [30].

By studying the optimized EKF algorithm (EKF compensator), it has been observed that the treatment is based on an elementary Dot-Product function (relation 11) that is used several times. Then, the $\mathrm{A}^{3}$ methodology can be applied.

$o(t)=x_{1}(t) \cdot y_{1}(t)+x_{2}(t) \cdot y_{2}(t)+x_{3}(t) \cdot y_{3}(t)$ 


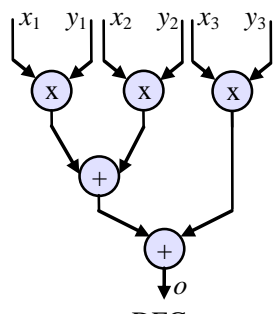

a - DFG

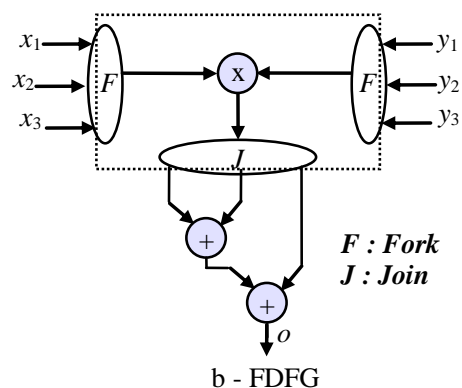

b - FDFG
Fig. 12. Developed DFG and FDFG

Fig. 12(a) shows the DFG of the developed Dot-Product. The multiplications can be performed in parallel mode which is not the case of additions. Thus, the factorization process can be applied to the multiplier operator and the obtained FDFG is presented in Fig. 12(b). Since this function is used several times, the EKF compensator can also be factorized. The obtained FDFG is then presented in Fig. 13 .

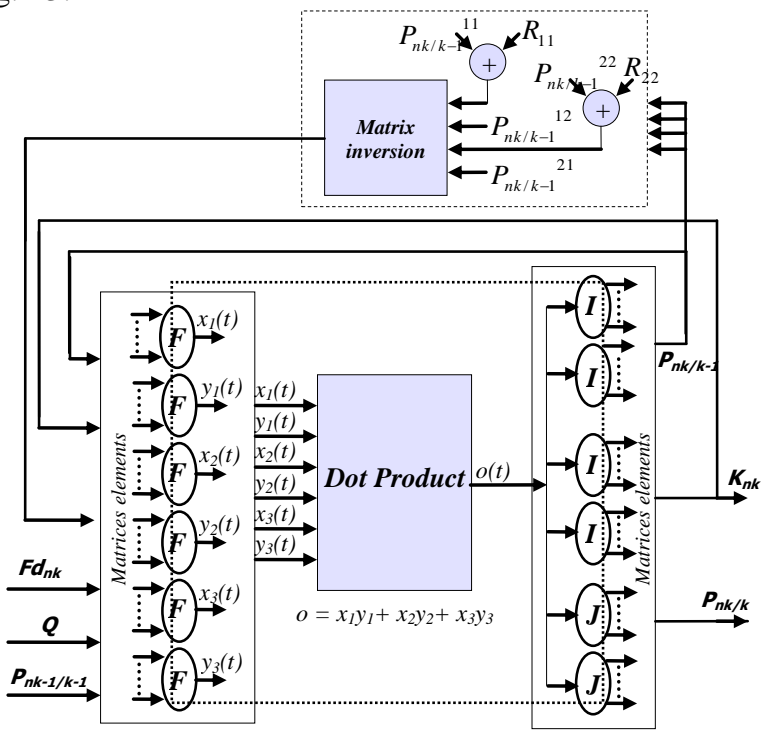

Fig. 13. Developed FDFG - Factorization of a thicker grain operator

2-Architecture design

According to the obtained FDFG, the FPGA-based architecture is designed by replacing the FDFG nodes $(\mathrm{F}, \mathrm{J}$ and I) by their corresponding operators. Thus, the node $\mathrm{F}$ is replaced by a multiplexer, J and I replaced by registers. The hardware architecture of each of the developed modules (according to the adopted partitioning) is then composed of a data path and a control unit that are both synchronized with the global clock signal.

The data path contains the used operators and data buses between them. The treatment scheduling is ensured by the control unit which is a simple Finite State Machine (FSM). The latter is activated via a Start pulse signal. When the computation time process is over, an End pulse signal indicates the end of the treatment. As an example, Fig. 14 presents the FPGA architecture corresponding to the FDFG of Fig. 12(b).

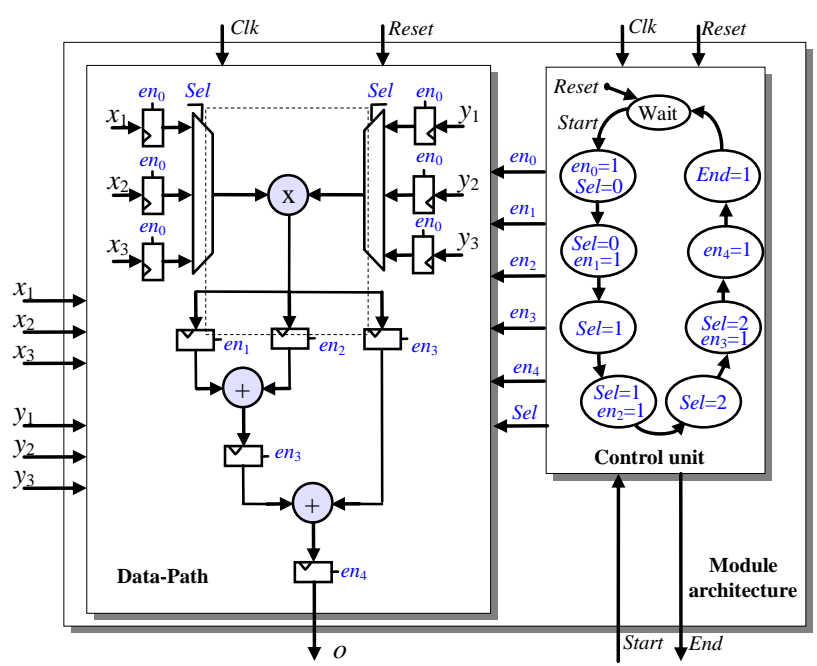

Fig. 14. Example of a designed FPGA architecture

\section{3- Functional simulation}

After having developed the VHDL design, the simulation is started. In our case, ModelSim tools have been used. The obtained simulation results can also be compared to those obtained during the Matlab/Simulink environment. Fig. 15 shows the obtained results for the presented application.

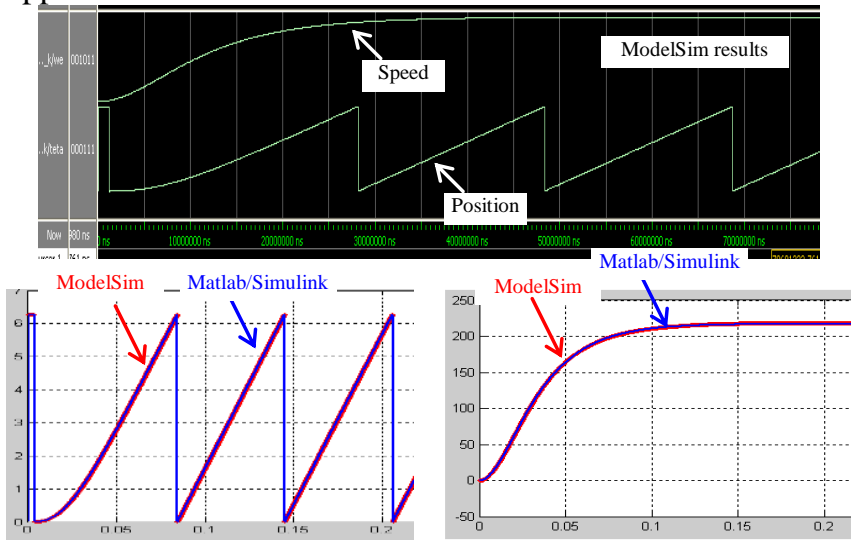

Fig. 15. Functional validation of the FPGA-based sensorless controller

4- Design synthesis and time/area performances analysis

Once the global FPGA architecture is functionally validated, the next step is the analysis of the time/area performances. This is obtained after having synthesized the developed design (using the dedicated synthesis tools). This synthesis indicates the consumed FPGA resources and the maximum frequency of the operating clock. This maximum frequency allows the calculation of the global execution time

Table VI summarizes the obtained synthesis results of the whole sensorless controller (including the EKF, speed and currents regulators). From these results, the time/area performances are extracted. The minimum execution time is given. All these data are listed for different FPGA device solutions including low cost and high performance Xilinx FPGAs.

Table VI: Synthesis results for the full FPGA-based sensorless controller

\begin{tabular}{|c|c|c|c|c|}
\hline & $\begin{array}{c}\text { Spartan 3E } \\
\text { xc3s } 1600 E\end{array}$ & $\begin{array}{l}\text { Spartan 6 } \\
\text { xc6slx150 }\end{array}$ & $\begin{array}{l}\text { Virtex 2P } \\
\text { xc2vp30 }\end{array}$ & $\begin{array}{c}\text { Virtex 6 } \\
\text { Xc6vsx475 }\end{array}$ \\
\hline $\begin{array}{c}\text { Max. clk } \\
\text { Frequency }\end{array}$ & $54 \mathrm{MHz}$ & $97 \mathrm{MHz}$ & $167 \mathrm{MHz}$ & $253 \mathrm{MHz}$ \\
\hline $\begin{array}{c}\text { Global } \\
\text { resources } \\
\text { use }\end{array}$ & $\begin{array}{c}\mathbf{- 4 5 \%}(3688 \\
\text { CLB) } \\
\mathbf{- 1 0 0 \%} \text { hw } \\
\text { 18-bit } \\
\text { multipliers }\end{array}$ & $\begin{array}{c}\mathbf{5 . 8 \%}(11519 \\
\text { CLB) } \\
\mathbf{- 2 6} \% \text { hw 18- } \\
\text { bit DSP } \\
\text { blocks }\end{array}$ & $\begin{array}{c}\mathbf{- 3 9 \%}(3424 \\
\text { CLB) } \\
\mathbf{- 3 8 \%} \text { hw 18- } \\
\text { bit multipliers }\end{array}$ & $\begin{array}{c}\mathbf{- 2 . 5 \%} \\
(37200 \\
\text { CLB) } \\
\mathbf{- 2 . 5 \%} \text { hw } \\
\text { 18-bit DSP } \\
\text { blocks }\end{array}$ \\
\hline
\end{tabular}




\begin{tabular}{|c|c|c|c|c|}
\hline$t_{\text {ex_min }}$ & $5,130 \mu \mathrm{s}$ & $3,214 \mu \mathrm{s}$ & $2,873 \mu \mathrm{s}$ & $2,712 \mu \mathrm{s}$ \\
\hline
\end{tabular}

D. Experimentation

\section{1- Hardware In the Loop (HIL) validation}

In order to verify a first operating attempt, it is highly recommended to start the experimentation by the HIL validation. The latter can be considered as an intermediate between a fully computer-based development validation (simulation tools and FPGA design tools) and a fully experimental validation (actual system platform). The HIL procedure is carried out through a physical implementation of the developed FPGA-based architecture to be validated. The latter has to be associated with a real-time emulation of the plant. In addition, a communication controller has to be implemented in order to transfer the stimuli and the probed data. This communication is made with a Host-PC in which a comparison between the obtained HIL results and the simulation results is made.

The developed EKF-based sensorless controller has been implemented and associated with an emulated plant model (synchronous motor model, VSI model and the mechanical load model). Fig. 16 highlights the synopsis of the achieved HIL test.

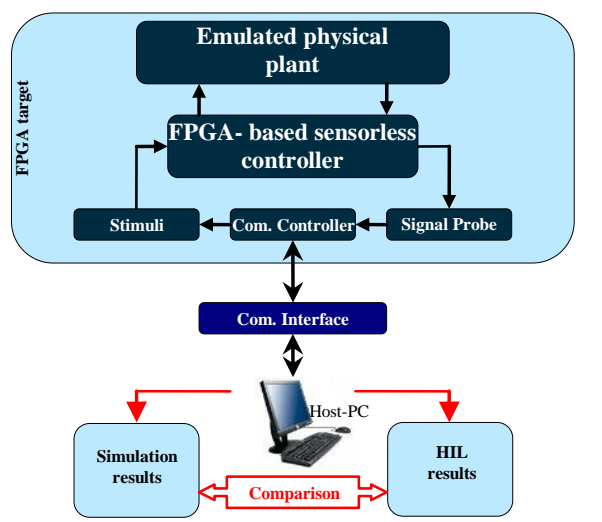

Fig. 16. HIL procedure

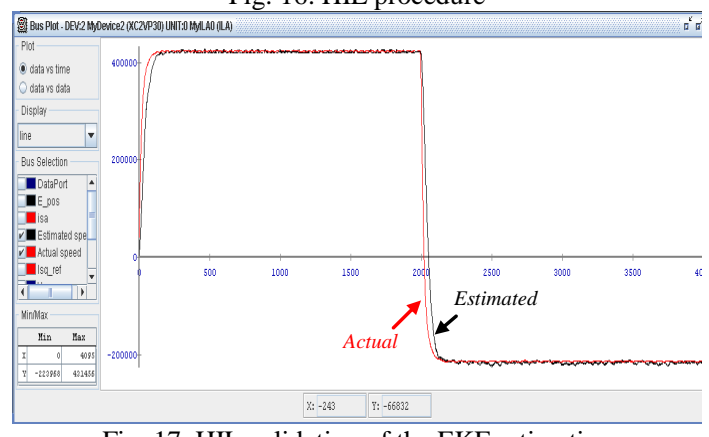

Fig. 17. HIL validation of the EKF estimation

When using a Xilinx FPGA target, the HIL procedure is made using the ChipScope analyzer [39]. The latter is used to probe the internal signals in one hand and to configure the design in the other hand. The data transfer is made using the JTAG interface. The obtained results are depicted in Fig. 17. The validation is then made by comparing them to the simulation results (Fig. 11 and Fig. 15).

\section{2- Experimental validation}

The experimental validation has been achieved with the platform presented in Fig. 18. For experimental constraints, the used digital control unit is based on a Xilinx XUP Virtex_2P board. The treatment is synchronized with a 50 $\mathrm{MHz}$ clock signal. The total execution time is then equal to $6 \mu \mathrm{s}$. Fig. 19 presents the waveforms of the speed and position estimation. These experiments are carried out with a $800 \mathrm{~W}$ four poles synchronous machine.
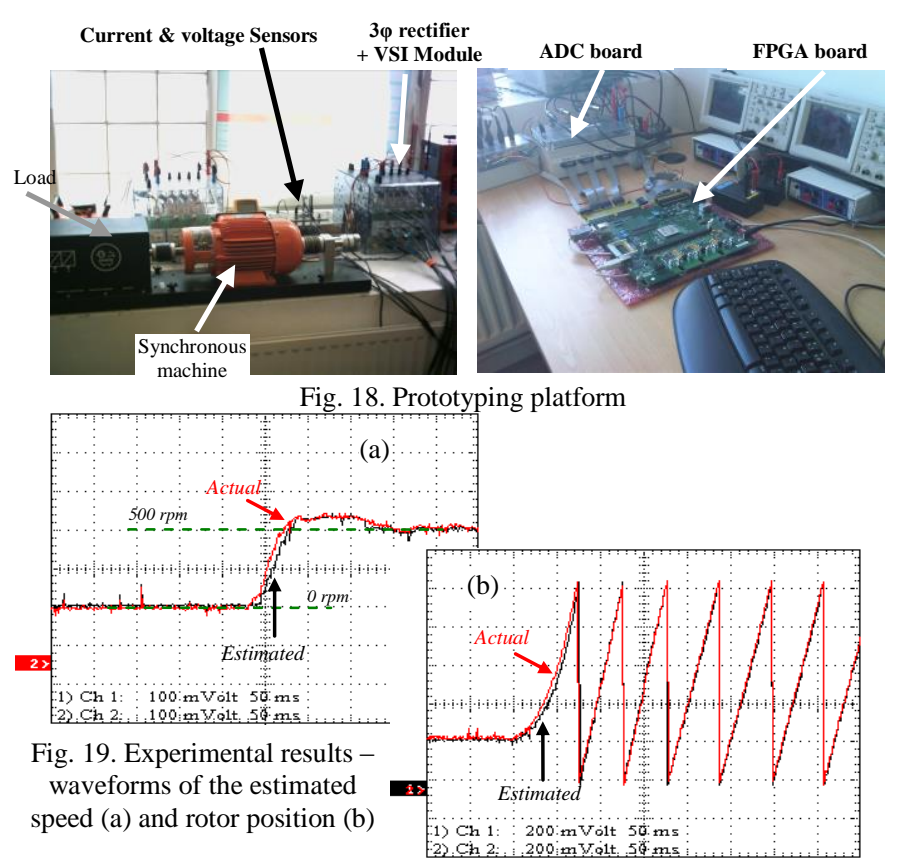

E. System on Chip FPGA design

For benchmarking reasons, we have also implemented the sensorless controller in software based on the synthesizable MicroBlaze [39] processor core. The Peripheral Local Bus (PLB) is used to connect this processor with the used peripherals (GPIO, timers, PWM, Interrupt controllers, user-defined data acquisition module...).

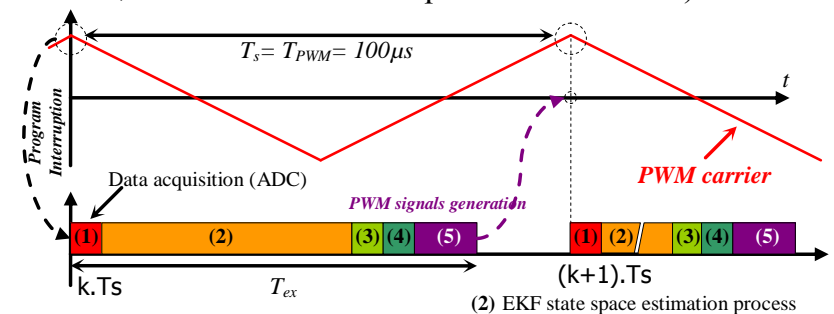

Fig. 20. Timing diagram - case of a full (3) P-PI speed controlle software sensorless controller $\quad$ (4) PI current controlle (5) PWM process

This software design is fully carried out using assembly macros and $\mathrm{C}$-coded functions. To augment the ALU performances of these functions, the optional "hardware multiply" and "hardware divide" are included. The EDK tool automatically generates the memory map of the hardware platform and assigns default drivers to the processor and each of its peripherals. The execution routine of the proposed algorithm is depicted in Fig. 20.

The MicroBlaze is synchronized with a $100 \mathrm{MHz}$ clock and the sampling period (interruption cycle) is set to $100 \mu \mathrm{s}$. The execution time of the whole sensorless controller has been evaluated to $85 \mu \mathrm{s}$. The surface occupation of the design is $11 \%$ of the used Virtex 5 XC5VLX50T FPGA. Note that external 12-bit ADCs are used for data acquisition. In order to make a comparison to a standard software solution, the same sensorless controller has been implemented in a TI TMSF2808 DSP device $(100 \mathrm{MHz}$, 32Bit, 12-bit ADC, 2x16-bit multiplier, 16Ko RAM memory, [63]). The same execution routine is preserved and the total execution time has been evaluated to $66 \mu \mathrm{s}$ [61]. The functions are fully $\mathrm{C}$-coded. One can stress from the obtained timing results that standard software devices remain more adapted for a full software implementation. This is mainly due to the design tools that are more mature. 
The way of coding and the quality of the compilation tools are also determinant points.

For these reasons and in order to benefit from the flexibility of SoC approaches and the high control performances achievable with hardware designs, it is interesting to combine both software and hardware treatments. For this aim, it is highly recommended to adopt co-design methodologies so as to make an intelligent partitioning between the software treatment and the hardware treatment.

\section{IV- FPGA CONTROLLERS BASED ON ARTIFICIAL INTELLIGENCE}

Traditionally, mathematical models were developed to evaluate the functionality of global engineering systems. However, the practical development of each part of the system needs then to be separately addressed. This often involves the use of different software platforms, the design itself being developed in a different environment. Recent advances in CAD tools have brought the functional description of design and practical hardware implementation closer.

The use of modern Electronic Design Automation packages for electronic systems design facilitates easy implementation of complex control algorithms and Artificial Intelligence (AI) into hardware. Hence, a wide range of complex and intelligent controller designs have been recently developed, with applications in industry [66-70]. A significant number of them target FPGAs, due to the rapid prototyping features and the flexibility offered by FPGAs, especially through the recent availability of microprocessor or DSP cores, allowing hardware software co-design and implementation [71].

A relevant paper in this context deals with custom architectures for Fuzzy and Neural Networks controllers [72]. It presents efficient architecture approaches to develop controllers using specific circuits, using HDLs and synthesizing them to get the FPGA configuration bit-stream. Some more specific areas using FPGAs for the implementation of complex controllers based on Artificial Intelligence are highlighted below.

\section{A. Neural Networks implemented in FPGA}

According to an European Network of Excellence (ENE) report [73], the future implementation of hardware neural networks is shaped in 3 ways: i) by developing advanced techniques for mapping neural networks onto FPGA, ii) by developing innovative learning algorithms which are hardware-realizable, iii) by defining high-level descriptions of the neural algorithms in an industry standard to allow full simulations and fabrication and to produce demonstrators of the technology for industry. Such new designs will be of use to industry if the cost of adopting them is sufficiently low. Hardware-based neural networks are important to industry as they offer low power consumption and small size compared to PC software and they can be embedded in a wide range of systems. Software libraries exist for traditional Artificial Neural Network (ANN) models (Matlab). The industry-standard form is however VHDL/Verilog or $\mathrm{C}++$ parameterized modular code, allowing customization.

A wide range of research papers on Neural Networks based Controllers were published in prestigious journals.
Some were collated in special issues on Transactions of Industrial Electronics [74, 75].

An interesting trend, which confirms the ENE report [73], is given by the recent development and implementation results of a parameterized FPGA-based architecture for feedforward multi layer perceptrons with backpropagation learning algorithm (FF-MLPs) presented in [76]. The proposed architecture makes possible native prototyping and design space exploration in hardware.

Others papers $[77,78]$ exploit the learn ability of ANNs. Paper [76] presents an adaptive low-speed-damping controller for a stepper motor which removes nonlinear disturbance at low speeds and paper [78] shows a self-tuning PID FPGA-based motion controller using RBF NN for a XY table.

\section{B. Fuzzy Logic Based Control Systems}

Today, fuzzy logic based control systems, or simply, Fuzzy Logic Controllers (FLCs) can be found in a growing number of products, from washing machines to speedboats, from air condition units to hand-held autofocus cameras. The success of fuzzy logic controllers is mainly due to their ability to cope with knowledge represented in a linguistic form instead of representation in the conventional mathematical framework. Control engineers have traditionally relied on mathematical models for their designs. However, the more complex a system, the less effective the mathematical model.

FPGAs constitute an appropriate target for the implementation of fuzzy-logic controllers, facilitated by the flexibility of the design environment, enabling direct implementation of the circuit's abstract model. A high number of works have been published on fuzzy logic-based control systems. One paper presents a method employing hardware/software co-design techniques according to an 'a priori' partition of the tasks assigned to the selected components. This feature makes it possible to tackle the control system prototyping as one of the design stages. In this case, the platform considered for prototyping has been a development board containing a standard microcontroller and an FPGA. Experimental results from an actual control application validate the efficiency of this methodology [79].

A paper, advocates a novel approach to implement the fuzzy logic controller for speed control of electric vehicle by using FPGA [80]. Another paper [81] presents an implementation of a FLC on a reconfigurable FPGA system, while a different paper explores the use of FPGA technologies to implement FLCs. Two different approaches are described. The first option is based on the logic synthesis of the boolean equations describing the controller inputoutput relations. The second approach uses dedicated hardware to implement the fuzzy algorithm according to a specific architecture based on a VHDL cell library [82]. A FPGA based fuzzy sliding-mode controller, which combines both the merits of fuzzy control and sliding-mode control, is proposed in [83], to control the mover position of a linear induction motor (LIM) drive to compensate the uncertainties including the frictional force.

Paper [84] presents an H-bridge multilevel converter governed by an integrated fuzzy logic controller/modulator designed with VHDL and implemented in FPGA.

A design environment for the synthesis of embedded FLC on FPGAs, which provides a novel implementation technique has been developed in [85]. It allows accelerating 
the exploration of the design space of fuzzy control modules, as well as a codesign flow that eases their integration into complex control systems. Even an FLC based ant colony optimization (ACO) application has been proposed in [86] for improving designs efficiency and control performance, as well as ACO hardware implementation.

\section{Intelligent Data Acquisition Devices (DAQ)}

Intelligent DAQ devices use National Instruments (NI) LabVIEW reconfigurable FPGAs to implement custom high-performance data acquisition on commercial off-theshelf (COTS) hardware. Instead of a predefined subset of DAQ functionality, the intelligent DAQ uses an FPGAbased system timing controller to make all analog and digital I/Os configurable for application-specific operation. By programming the FPGA, the custom high-performance DAQ tasks can easily be implemented. Additionally, because of the parallel architectures of FPGAs, the highperformance task implementation is achieved without performance degradation [87]. DMA provides a direct link for data on the FPGA to RAM on the host machine, improving data-logging efficiency and making data immediately available for analysis and visualization. This high-speed data transfer provides real-time visibility into parameters and variables within the FPGA.

Furthermore, adding an FPGA circuit to a DAQ platform interfaced by the NI Labview graphical system design tool gives the ability to perform early signal pre-processing and offers new valuable abilities like reconfigurability - first step towards a self reconfigurable device ready to respond in real time to any external or intrinsic changes [88]. On the other hand, Labview may be used as a graphical programming environment for FPGA targeted designs. The new LabVIEW FPGA environment enables application domain experts without prior knowledge of hardware description languages (HDLs) to program reconfigurable hardware devices. Such method is used in paper [89], illustrating how LabVIEW FPGA supports a flexible, reliable and cost-effective hardware design considering an ultra-high speed control application with complex timing synchronization. Other applications may be found in paper [90], in which an isolated wind energy conversion system based on the cascaded H-bridge multilevel inverter topology is considered or paper [91] which presents a high frequency radars controller designed in NI LabVIEW FPGA or even space applications as in paper [92]. A powerful combination of the parallel signal processing using neural networks implemented in FPGA with Labview as interface for an intelligent DAQ can be found in paper [93]. The output is an implementation of a neural network based estimator of the load machine speed for two-mass drive system on FPGA.

\section{Evolvable hardware}

Evolvable hardware offers much for the future of complex system design. Evolutionary techniques not only give the potential to explore larger solution spaces, but when implemented on hardware allow system designs to adapt to changes in the environment, including failures in system components. Evolutionary algorithms have been developed and applied to intrinsic hardware evolution, aiming to produce an evolutionary system that can be readily implemented on COTS hardware. An FPGA-based controller for a mobile robot has been developed by Prof. Andy Tyrrell and his team at University of York, UK. The controller consists of look-up tables, which perform the mapping from sensor data to actuator, evolved using an effective evolutionary algorithm [94]. Paper [95] presents how a self-generated architecture may be used to build-up a secret physical cipher unit with dynamic security properties. Another application can be found in [96], where a run-time adaptable evolvable hardware classifier system is proposed or in [97], in which a FPGA based customizable generalpurpose GA engine has been reported. A novel bio-inspired self-test technique for the implementation of evolvable fault tolerant systems, which mimics a Unitronic (unicellular electronic) artificial system, is presented in [98]. The system is implemented in FPGA like a bio-inspired cellular array and made up of structurally identical cells with selfdiagnostic and self-healing capability.

\section{V- NEURAL NETWORK BASED FPGA SYSTEMS - CASE STUDIES}

Artificial Neural Networks (ANNs) are also an interesting research field where FPGAs have been successfully used. Many recent publications ([9], [99], [100], [101]) consider the FPGA as an effective implementation solution of control algorithms for industrial applications. Hardware implemented ANNs have an important advantage over computer simulated ones by fully exploiting the parallel operation of the neurones, thereby achieving high speed of information processing.

A. Case study 1: FPGA NN Hardware Implementation Algorithm

Some VLSI algorithms achieve efficient NN implementation. An algorithm for compact neural network hardware implementation, by using a combination of AND gates, OR gates and Threshold Gates (TG), leads to compact hardware structures. However, it cannot be used for direct FPGA implementation because TGs are not available in FPGA logic cells [102]. In order to minimize both ASIC and FPGA hardware implementation of ANNs composed of neurones with step activation functions, the solution is to treat each neurone as a Boolean function and to implement it separately. This leads to minimize the implementation complexity. The most useful property of such a Boolean function is that if its truth table is constructed as a matrix with as many dimensions as neurone inputs, then the truth table has only one large group of ' 1 ' and one large group of ' 0 '. The solid group of ' 1 ' is not visible when the Gray codification is used and thus classical Quine-McClusky algorithms or Karnaugh maps cannot efficiently be used. The proposed algorithm [103] uses a different approach and generates a multilayer pyramidal hardware structure, where layers of AND gates alternate with layers of OR gates. The bottom layer consists of incomplete NOT gates, a structure to be optimised later by eliminating redundant logic gates groups.

However, the method is effective only when the numbers of inputs and bits on each input are low, otherwise a classical circuit may be more efficient. The algorithm itself contains three steps: ANN mathematical model digitisation, conversion of the digitised model into a logic gate structure, and hardware optimisation by elimination of redundant logic gates. A set of $\mathrm{C}++$ programs automates algorithm implementation, generating optimised VHDL code. This strategy bridges the gap between ANN design software and hardware design packages (Xilinx). Although the method is 
directly applicable only to neurones with step activation functions, it can be extended to sigmoidal functions.

The sample in Fig. 21 shows a neurone with 12 input weights and positive threshold level. The weights are sorted in descending order and a recursive implementation starts. The first three weights are larger than the threshold, so inputs 4, 7, 1 will drive an OR gate along with the subneurones built using the other subgroups [104]. The algorithm was automated using $\mathrm{C}++$ programs that generate a netlist description of the circuit, optimize it and then generate the VHDL code. In terms of the software, there is no limitation of the ANN size. The characteristics of the ANN are introduced in the $\mathrm{C}++$ program as a matrix text file (.csv format). Thus, a feed forward ANN with 3 subnetworks (Fig. 22), generating the PWM switching pattern for an inverter, was designed [103].

In contrast with training algorithms, constructive ones determine both the network architecture and the neurone weights and are guaranteed to converge in finite time. The numerical values of all neurone weights and thresholds were calculated using a geometric constructive solution known as Voronoi diagrams. For this work, the complex plane is divided into triangular Voronoi cells. The master program allows user control over main parameters: i) Number of Voronoi cells, ii) Number of sectors dividing the 360 degrees interval for argument analysis, iii) Number of bits used to code the components of the two complex inputs iv) Maximum fan-in for the VHDL logic gate model. The desired performance / complexity ratio is adopted. In this case, 5 bits to code each component of the two complex inputs gives enough precision (delays less than $100 \mathrm{~ns}$ ), resulting in a total number of logic gates of 1329 on 14+6=20 layers, which fits Xilinx XC4010XL FPGA.

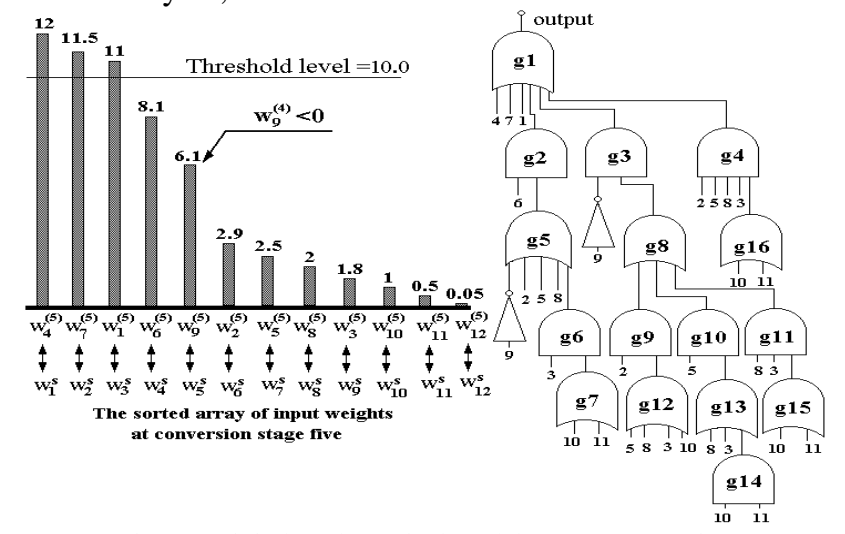

Fig. 21: Digital mathematical model to gate conversion

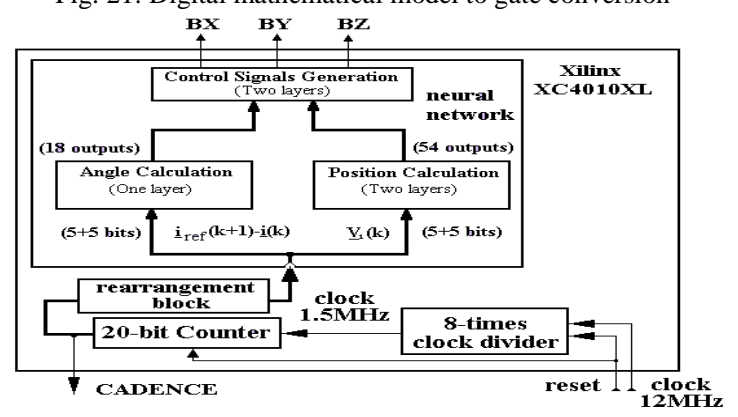

Fig. 22: ANN structure and testbench for operation speed testing

Angle: analyses the argument of current difference vector.

Position: analyses the argument and value of the voltage.

Control Signals: generates three PWM binary outputs.

When the number of inputs and bits on each input is low (precision appropriate for drives), this method is more effective than a classical digital circuit design implemented in FPGA. For a high number of bits/controller inputs, the $\mathrm{NN}$ approach can be less effective than a classical circuit. The explanation is that in the NN approach the complexity of the resulting circuit raises exponentially with these numbers, whereas in a traditional approach, the complexity increases quadratically. The case study presented in this paper was implemented as part of an induction motor controller in a 10,000 gates equivalent FPGA, as opposed to a classical digital vector control circuit, for controlling the same motor, which uses $99 \%$ of a 40,000 gate equivalent FPGA [105].

\section{B. Case study 2: FPGA NN based electronic nose}

Another interesting example is related to neural networks design applied to an FPGA based artificial olfactory system [106]. The method presented leads to the conceptual development of an extendable hardware implementable neural library of a feed-forward back propagation network (FF-BP) with on-chip learning. Neural modules, which can emulate in hardware the FF-BP computing functions, are grouped into a neural library and can in principle be used to create any FF-BP NN topology by setting the NN characteristics as number of neurons and layers. The case study shows an ANN used as a pattern recognition module in an artificial olfaction system, which is capable to identify four coffee brands [107]. An extended analysis has been carried out regarding the recognition rates versus training data features and data representation.

The adopted approach replaces the classical solution of defining the application by using a high level language, it will be more useful and user friendly to create a pattern recognition system, in our case an ANN, by means of configurable modules grouped into a specific library. The immediate advantage of this approach is given by the possibility to implement different ANNs in hardware in the early research phases of the ANN development. Also, it gives the opportunity to establish, through simulation, the best resolution for data representation (Fig. 23). The research was carried out on developing a neural library set for a Feed-Forward Back Propagation (FF-BP) topology. The library is designed to support on-chip learning, giving therefore premises of self configuration capabilities in terms of pruning or enlarging the neural network's size in order to obtain a fully optimized neural network for a specific application. The results are used to develop an intelligent platform with learning and adaptive properties. Building such a platform presumes the creation of predefined libraries of different Artificial Neural Networks (ANN) components, which finally may be used to create any neural network.

A system for identifying different olfactory chemical compounds (4 different coffee brands) was developed as application [106]. The system recognizes different patterns (olfactory signature of organic molecules) using neural networks as pattern recognition, hardware implementable in programmable logic circuits such as FPGA. 


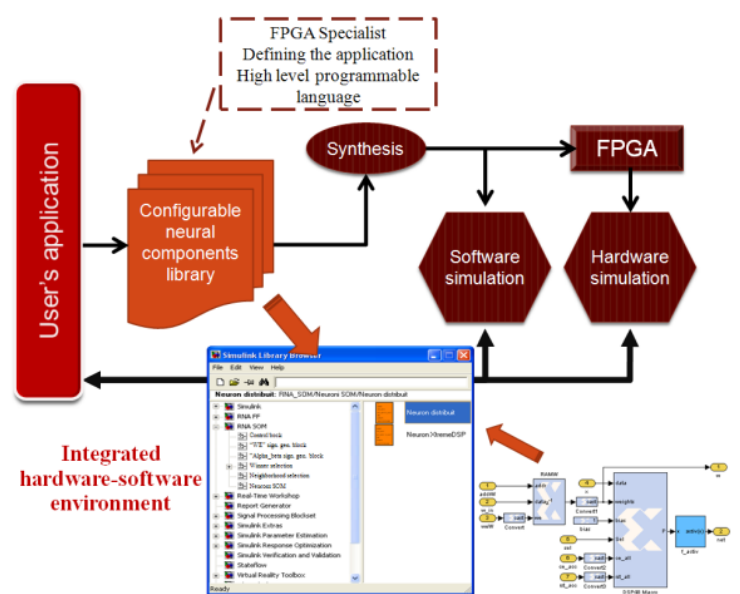

Fig. 23. Proposed method for hardware implemented ANN design

Discovering the optimal topology of an ANN for a particular application remains an important issue, especially for highly competent classifiers such as FF-BP neural networks. It most cases, so far, the appropriate choice of the network features, the training methodology to be used, and the best network topology have been identified by repetitive simulations and modifications of the project code. Therefore, a library with ANN components, ready to be hardware implemented, represents a step forward towards a more user friendly design environment. An extendable hardware implementable neural library has been developed, considering a FF-BP network topology with on-chip learning. The implementation goal is achieved using the Mathworks' Simulink environment for functional specification and System Generation, to generate the VHDL code according to the chosen FPGA device features.

In order to discuss the functions that have to be implemented, it is important to specify the architecture of the MLP networks: the neurons are organized in at least 3 layers: one input layer, one output layer and one or more intermediate, hidden layers. The network is fully connected, i.e. all the outputs of a layer are connected by synapses to all inputs of the following layer. Only the hidden and the output layers include processing units, whereas the input layer is used just for data feeding. The parallelism adopted is a node one and requires managing all the neurons from the same layer at the same time. Therefore, the control blocks that command the behaviour of the neuron elements are common for all neurons of a layer.

Another main component of the artificial neuron, and usually a bottleneck for its speed performance, is the sigmoid activation function. Its hardware implementation implies important hardware resource utilization [108]. In order to reduce such consumption is useful to adopt different approximations (depending of the available hardware resources) with minimum errors. The principal classical methods to digitally implement an activation function are Look-up tables and truncation of the Taylor series expansion. Also, there are approximations with smaller errors, but they use floating-point multiplications, thus a practical VLSI implementation becomes far too complicated $[109,110]$.

In order to create the library modules of the sigmoid firing function, using System Generator/Simulink blocks, hardware implementations for the following approximation functions have been produced. The implementation reports conclude that that the best approximation method is the
PLAN function, when the number of the neurons that use sigmoid function as fire function is larger than the number of the BRAM blocks available in the FPGA circuit. When the number of neurons is lower than the total BRAM blocks available in the FPGA circuit, the best way to approximate the sigmoid function is the Lookup Tables method.

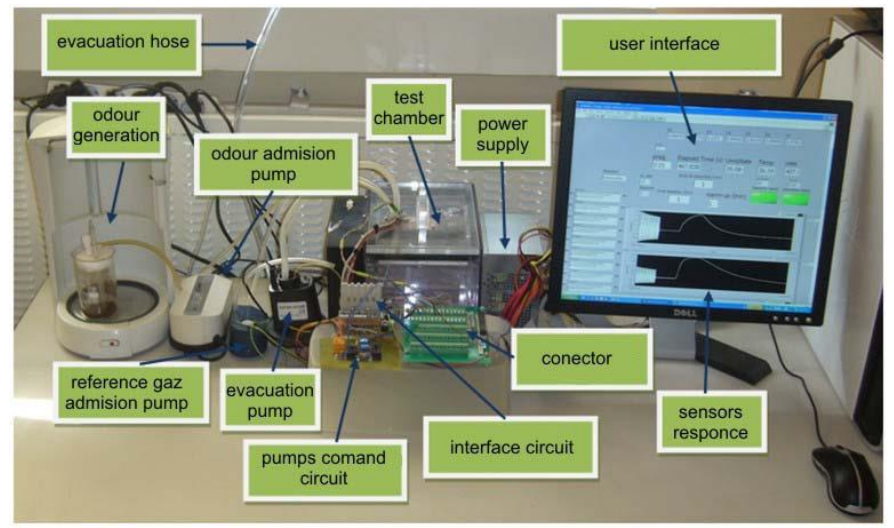

Fig. 24. The architecture of the adopted artificial olfaction system

The process parallelism adopted implies that all the neurons within the same layer are controlled at the same time. The blocks are described using VHDL language and implemented using a block that converts a VHDL design into a System Generator/Simulink block. The computation results show that the largest NN which can be implemented into the 4VSX35 circuit is a FF-BP neural network with maximum of 120 neurons distributed as: 80 in the hidden layer and 40 in the output layer.

The FF-BP neural network design environment developed was used to create a pattern recognition module for an artificial olfactory system capable to recognize different types of coffee. The olfactory system consists of (Fig. 24):

- seven gas sensors chosen to react to a wide spectrum of odours (TGS842, TGS826_1, TGS826_2, TGS2600, TGS2601, TGS2602, TGS2620),

- temperature sensor (LM35),

- humidity sensor (SY-HS-230) - (all sensors are mounted into a gas test chamber),

- test chamber,

- three pumps for gas carrying,

- circuits for sensors conditioning and pumps command,

- data acquisition board (PCI-MIO-16E-1),

- pattern recognizing module hardware implemented in FPGA (Virtex-4 SX MB - 4VSX35),

- user interface developed in Labview 8.2.

The data acquisition module was created in Labview environment using a virtual instrument (VI) customized to execute the following tasks: to acquire data generated by all 9 sensors, to pre-process the acquisitioned signals (filtering, drift cancellation) and to save data as text file format (.lvm file). Therefore, the modules that compound the VI consist of: pumps control module, data acquisition control module, sub-VIs control time module, pre-processing signal module, $\mathrm{C}$ grade conversion module of the signal generated by the temperature sensor and \% RH conversion module of the signal generated by the humidity sensor. 


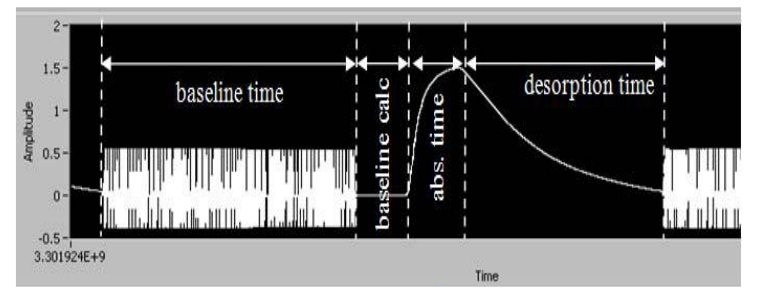

Fig. 25. Voltage variation with baseline time, baseline calculation time, absorption time and desorption time

The data has been extracted using the scenario presented in Fig. 25. It roughly comprises three processes. The first is the baseline calculation (the average voltage drop on sensors resistance when the reference gas, air, is applied). The second and third stages presume measurement with a fixed sampled frequency over a defined time, absorption and respectively desorption time, of the voltage drop on sensors resistance when the enriched odour is applied. A regular absorption / desorption operation, which presents the results of time settings vs. voltage drop on sensor resistor is shown in Fig. 25.

To conclude the second case study presented, it can be stated that a novel neural design strategy has been developed, which benefits of reduced design time over classical field orientation approaches, leading to a low complexity and easy to implement pattern recognition module. A particular application of the pattern recognition system for an olfactory system was investigated and results presented show efficient hardware implementation in FPGA circuit. The neural network used as pattern recognition system was designed using a hardware-software co-design environment, in a manner that facilitates control over the hardware implementation complexity, and was downloaded into a Xilinx FPGA circuit.

The achievement presented in this case study refers to a holistic modelling/design method, using modules created into hardware-software co-design environment (MatlabSystem Generator-ISE) and grouped in a specific NN library. These modules emulate in hardware any FF-BP network topology behaviour, giving the opportunity to design hardware implementable FF-BP neural networks, at a higher level. As a specific application, a FF-BP network has been used as pattern recognition module for an artificial olfactory system, capable to recognize 4 coffee types. Also, the influence over the recognition rates of data representation resolution and data training dimensionality has been analysed. The observations have been used to determine the neural network which has the best recognition rates with minimum hardware resource utilization.

\section{V-CONCLUSIONS AND FUTURE TRENDS}

The aim of this paper was to present the FPGA technologies and highlight their use in industrial control applications. It has been underlined that these hardware solutions can perfectly address the nowadays challenges in this field such as high control performances, low cost, reliability, power consumption and immunity to radiations.

The contribution of these hardware devices was then discussed. The case of embedded systems, and industrial control systems were focused on. For the embedded systems, it has been stated that FPGAs are becoming an interesting option in many applications like robotics, networking, automotive and aircraft. Indeed, FPGAs are bringing many benefits in terms of safety, rapidity and power consumption.

In the case of control applications, It has been shown that FPGA-based controller can be an efficient option for both the high demanding applications and the constrained switching frequency applications. The first group corresponds to high switching frequency applications, high level of parallelism applications and HIL applications. Regarding the second group for which the switching frequency is limited, it has been shown that we can take advantage of the rapidity of FPGAs to boost the performances of the controller.

Authors have firstly made a generic presentation of the FPGAs. Their internal structure and their configuration technologies were described. A discussion about the design tools was also made.

In the industrial control domain, the control algorithms become more and more complex. To illustrate the interest of FPGAs in such complex controller, authors have presented an example of application which consists of a sensorless motor controller. To estimate the rotor position and the speed of the used synchronous machine, an Extended Kalman Filter was used. The development of the FPGAbased sensorless controller was then made according to a design methodology which is required especially for such complex algorithms.

Another relevant field which was discussed is the Neural Network (NN) systems. The use of FPGAs in such applications is highly interesting since the parallelism of the NN algorithms is preserved. For an illustration purpose, authors have resented case studies.

In the near future, the complexity of the digital control systems will continue to grow and the tasks devoted to the control algorithm will no longer be limited to regulation but have to manage EMI, communications, health monitoring, diagnosis and fault-adaptive on line control. As a consequence and from an algorithm point of view, signal processing functions for power applications will be the center of intensive researches. From this perspective, FPGAbased controller represents a very attractive solution too.

Finally, we shall end by shortly introducing the main challenges in terms of architecture, this aspect being closely related to the algorithm and at the same time offers in itself a degree of creativity. Among the most exciting trends we can quote the introduction of the floating point computing evaluation for FPGA-based solutions. What is its cost in terms of hardware resources? Is it necessary for industrial control applications where the dynamic range of variables and the complexity of the control algorithm are still limited? Another interesting field of research is the co-design approach, with questions like how to make an optimized partitioning between the software part of the controller architecture and its hardware part?, [111]. Another issue concerns mixed integration. Indeed, as seen before and due to their rapidity FPGA-based controllers are now introducing only a small delay in the loop and because of their high density rate can easily work with a high accuracy (high number of bits). This makes the controller quasitransparent moving the limitations of time and accuracy towards the interfaces like ADCs and the drivers.

Another challenging point is the system integration. Indeed, in embedded systems (e.g. aircraft, space, automotive) the surface occupation of the systems is a key issue. To this purpose, it is advantageous to used digital 
solutions that ensure a high level of integration. Here again, the use of FPGA SoC devices is quite promising. Along the trend, novel device technologies that also integrate mixed analog/digital features in the same device are now available.

Finally, we shall finish this enumeration by reminding that SRAM-based FPGA architecture can be reconfigured on the fly. This feature has also to be explored having in mind fault detection and reconfiguration controller or optimized architecture [112]. In this case, the choice of the operating system is crucial.

\section{REFERENCES}

[1] Ajay V Deshmukh, "Microcontrollers, Theory and Applications", the MacGraw-Hill companies, Computer Engineering series, 2007 Phil Lapsley, "DSP processor fundamentals: architecture and features", IEEE Press, Computers, 1997

[3] Wayne Hendrix Wolf, "FPGA-based system design", Prentice Hall PTR, 2004

[4] Actel on-line literature. Available in www.actel.com

[5] E. Monmasson, M. Cirstea, "Guest Editorial special section on field programmable gate arrays (FPGAs) used in industrial control systems", IEEE Trans. On Ind. Electron., vol. 54, no. 4, pp. 18071809, August 2007

[6] E. Monmasson, M. Cirstea, "Guest Editorial special section on field programmable gate arrays (FPGAs) used in industrial control systems", IEEE Trans. On Ind. Electron., vol. 55, no. 4, pp. 1499 1500, April 2008

[7] J. Munoz-Castaner, R. Asorey-Cacheda, F.J. Gil-Castineira, F.J. Gonzalez-Castano, P.S. Rodriguez-Hernandez, "A Review of Aeronautical Electronics and its Parallelism with Automotive Electronics", IEEE Trans. On Ind. Electron., vol. 54, no. 99, April 2010

[8] P. Conmy, I. Bate, "Component-Based Safety Analysis of FPGAs", IEEE Trans. On Ind. Informatics, Vol.6, n² 2, pp. 195, May 2010

[9] F. Salewski, S. Kowalewski, "Hardware/Software Design Considerations for Automotive Embedded Systems", IEEE Trans On Ind. Informatics, Vol. 4, ${ }^{\circ} 3$, pp. 56, August 2008

[10] H. Guzman-Miranda, L. Sterpone, M. Violante, M.A. Aguirre, M. Gutierrez-Rizo, "Coping With the Obsolescence of Safety- or Mission-Critical Embedded Systems Using FPGAs", IEEE Trans. On Ind. Electron., vol. 58, no. 3, pp. 814, Marsh 2011

[11] M.A. Gora, A. Maiti, P. Schaumont, "Coping With the Obsolescence of Safety- or Mission-Critical Embedded Systems Using FPGAs", IEEE Trans. On Ind. Informatics, Vol. 6, n ${ }^{\circ}$, pp. 719, Nov. 2010

[12] F. Simonot-Lion, "Guest Editorial Special Section on In-Vehicle Embedded Systems", IEEE Trans. on Ind. Informatics, vol. 56, no. 4, pp. 372 - 374, Oct 2009.

[13] F. Baronti, E. Petri, S. Saponara, L. Fanucci, R. Roncella, R. Saletti, P. D'Abramo, R. Serventi, "Design and Verification of Hardware Building Blocks for High-Speed and Fault-Tolerant InVehicle Networks", IEEE Trans. On Ind. Electron., vol. 58, no. 3 , pp. 792, Marsh 2011

[14] J.-J. Chen, X. S. Hu, Mossé, D. , L. Thiele, "Guest Editorial Special Section on Power-Aware Computing ," IEEE Trans. on Ind. Informatics, vol. 57, no. 3, pp. , July 2010.

[15] J. Choi, H. Cha, "A Processor Power Management Scheme for Handheld Systems Considering Off-Chip Contributions ," IEEE Trans. on Ind. Informatics, vol. 57, no. 3, pp. , Jyly 2010.

[16] Fuming Sun, Haiyang Wang, Fei Fu, Xiaoying Li, "Survey of FPGA low power design”, In Proc. ICICIP'2010 Conf., pp. 547 550,2010

[17] P. Ferrari, A. Flammini, D. Marioli, A. Taroni, "A Distributed Instrument for Performance Analysis of Real-Time Ethernet Networks", IEEE Trans. On Ind. Informatics, Vol. 4, $\mathrm{N}^{\circ}$ 1, pp. 1625, Feb. 2008

[18] S. Fischmeister, R. Trausmuth, Insup Lee, "Hardware Acceleration for Conditional State-Based Communication Scheduling on RealTime Ethernet ," IEEE Trans. on Ind. Informatics, vol. 56, no. 3, pp. 325 - 337, July 2009.

[19] L. Vachhani, K. Sridharan, "Hardware-Efficient PredictionCorrection-Based Generalized-Voronoi-Diagram Construction and FPGA Implementation”, IEEE Trans. On Ind. Electron., vol. 55, no. 4, pp. 1558, April 2008

[20] L. Vachhani, K. Sridharan, P.K. Meher, "Efficient FPGA Realization of CORDIC With Application to Robotic Exploration", IEEE Trans. On Ind. Electron., vol. 56, no. 12, pp.
4915, Dec. 2009

[21] C.-C. Tsai, H.-C. Huang, S.-C. Lin, "FPGA-Based Parallel DNA Algorithm for Optimal Configurations of an Omnidirectional Mobile Service Robot Performing Fire Extinguishment", IEEE Trans. On Ind. Electron., vol. 58, no. 3, pp. 1016, March 2011

[22] H. Tanaka, K. Ohnishi, H. Nishi, T. Kawai, Y. Morikawa, S. Ozawa, T. Furukawa, "Implementation of Bilateral Control System Based on Acceleration Control Using FPGA for Multi-DOF Haptic Endoscopic Surgery Robot”, IEEE Trans. On Ind. Electron., vol. 56, no. 3, pp. 618, March 2009

[23] Li. Tianjian, Y. Fujimoto, "Control System With High-Speed and Real-Time Communication Links", IEEE Trans. On Ind. Electron., vol. 55, no. 4, pp. 1548, April 2008

[24] E. Monmasson, M. W. Naouar, L. Idkhajine, "FPGA-based Controllers for Power Electronics and Drive Applications", IEEE Ind. Electron. Magazine, vol. 5, n${ }^{\circ}$, pp. 1-13, March 2011

[25] O. Lopez,, J. Alvarez, J. Doval-Gandoy, F.D. Freijedo, "Multilevel Multiphase Space Vector PWM Algorithm" IEEE Trans. Ind Electron., vol. 55, no 5, pp 1933 - 1942, May 2008.

[26] B. J. Patella, A. Prodic, A. Zirger, and D. Maksimovic, "Highfrequency digital controller IC for dc-dc converters," IEEE Trans. Power Electron., vol. 18, no. 1, pp. 438-446, Jan. 2003.

[27] S.C. Huerta, A. de Castro, O. Garcia, J.A. Cobos, "FPGA-Based Digital Pulsewidth Modulator With Time Resolution Under 2 ns", IEEE Trans. on Power Electron., vol. 23, nº, pp. 3135-3141, Nov. 2008.

[28] A. V. Peterchev and S. R. Sanders, "Quantization resolution and limit cycling in digitally controlled PWM converters," IEEE Trans. Power Electron., vol. 18, no. 1, pp. 301-308, Jan. 2003.

[29] A. Myaing, V. Dinavahi, "FPGA-Based Real-Time Emulation of Power Electronic Systems With Detailed Representation of Device Characteristics," IEEE Trans. Ind. Electron., vol. 58, $\mathrm{N}^{\circ} 1$, Dec. 2010.

[30] M.-W. Naouar, E. Monmasson, A. A. Naassani, I. SlamaBelkhodja and N. Patin, "FPGA-based current controllers for AC machine drives-A review," IEEE Trans. Ind. Electron., vol. 54, no. 4, pp. 1907-1925, Aug. 2007

[31] S. Karimi, A. Gaillard,P. Poure, S. Saadate, S. "FPGA-Based Real-Time Power Converter Failure Diagnosis for Wind Energy Conversion Systems," IEEE Trans. Ind. Electron., vol. 55, n¹2, pp. 4299-4308, Dec. 2008

[32] F. Blaabjerg, P.C. Kjaer, P.O. Rasmussen, C. Cossar, "Improved digital current control methods in switched reluctance motor drives," IEEE Trans. on Power Electron., vol. 14, n³, pp. 563572, May 1999.

[33] K. Tazi, E. Monmasson and J.P. Louis, "Description of an entirely reconfigurable architecture dedicated to the current vector control of a set of AC machines," In Proc. IEEE-IECON'99 Conf., 1999, pp. $1415-1420$.

[34] Y.-S. Kung, R.-F. Fung, and T.-Y. Tai, "Realization of a motion control IC for $\mathrm{x}$-y table based on novel FPGA technology," IEEE Trans. on Ind. Electron., vol. 56, no. 1, Jan. 2009, pp. 43-53.

[35] A. Fratta, G. Griffero, and S. Nieddu, "Comparative analysis among DSP and FPGA-based control capabilities in PWM power converters," in Proc. IEEE IECON'04 Conf., 2004, pp.257-262.

[36] B. Miao, R. Zane, D. Maksimovic, "System identification of power converters with digital control through cross-correlation methods," IEEE Trans. on Power Electron., vol. 20, n5, pp. 1093109, Sept. 2005.

[37] A. Ordaz-Moreno, R. De Jesus Romero-Troncoso, J.A. Vite-Frias, J.R. Rivera-Gillen, A. Garcia-Perez, "Automatic Online Diagnosis Algorithm for Broken-Bar Detection on Induction Motors Based on Discret Wavelet Transform for FPGA Implementation," IEEE Trans. Ind. Electron., vol. 55, n5, pp. 2193-2202, May 2008.

[38] P. Simi Valsan and K. Shanti Swarup, "High-Speed Fault Classification in Power Lines: Theory and FPGA-Based Implementation," IEEE Trans. on Ind. Electron., vol. 56, no. 5, pp. 1793-1800, May 2009.

[39] Xilinx on-line literature. Available in www.xilinx.com

[40] Altera on-line literature. Available in www.altera.com

[41] J. J. Rodriguez-Andina, M. J. Moure, M. D. Valdes, "Features, design tools, and application domains of FPGAs", IEEE Trans. On Ind. Electron., vol. 54, no. 4, pp. 1810-1823, August 2007.

[42] E. Monmasson, M. Cirstea, "FPGA design methodology for industrial control systems - A review," IEEE Trans. On Ind. Electron., vol. 54, no. 4, pp. 1824-1842, August 2007.

[43] E. Monmasson, L. Idkhajine, I. Bahri, M.W. Naouar, L. Charaabi, "Design methodology and FPGA-based controllers for Power Electronics and drive applications", In Proc. ICIEA'2010 Conf., pp. 2328-2338, Taichung, Taiwan. 
[44] L.Idkhajine, E. Monmasson, M-W. Naouar, A.Prata and K.Bouallaga, "Fully integrated FPGA-based controller for synchronous motor drives," IEEE Trans. Ind. Electron., vol. 56, $\mathrm{n}^{\circ} .10$, pp. 4006-4017, Oct. 2009.

[45] K. Eshraghian, "SoC Emerging Technologies", IEEE Proceedings, vol. 94, no. 6, Jun. 2006, pp. 1197 - 1213

[46] G. Martin, "Overview of the MPSoC Design Challenge". in Proc DAC'06 Conf., 2006, CD-ROM.

[47] R. Obermaisser, P. Gutwenger,"Model-Based Development of MPSoCs with Support for Early Validation", in Proc. IEEE IECON'09 Conf., 2009, CD-ROM.

[48] R. Kumar, D.Tullsen, and N.Jouppi. «Core Architecture Optimization for Heterogeneous Chip Multiprocessors". In Proc. PACT'2006 Conf., Seattle, April 2006

[49] M. Youssef, S. Yoo, A. Sasongko, Y. Paviot, A. A. Jerraya, "Debugging HW/SW Interface for MPSoC: Video Encode System Design Case Study”, In Proc. DAC'04 Conf., 2004, CDROM.

[50] C. A. Zeferino, M. E. Kreutz, L. Carro, and A. A. Susin. "A study on communication issues for systems-on-chip," In Proc. SBCCI Conf., 2002, pp. 121-126.

[51] A. Brinkmann, J. Niemann, I. Hehemann, D. Langen, M Porrmann, and U. Ruckert. "On-chip interconnects for next generation systems-on-chips," in Proc. IEEE ASIC SOC Conf., 2002, pp. 212-215.

[52] P.P Pande, C. Grecu, M. Jones, A. Ivanov. "Performance Evaluation and Design Trade-Offs for Network-on-Chip Interconnect Architectures", IEEE Trans. On computers, vol. 54, n 8, pp. 1025-1040, Aug. 2005.

[53] J. J. Labrosse, MicroC/OS-II: The Real-Time Kernel. CMP Books, 2002.

[53] M. W. Naouar, A. A. Naassani, E. Monmasson, and I. SlamaBelkhodja. "FPGA-based predictive current for synchronous machine speed drive," IEEE Trans. Power Electron., vol. 23, no. 4, pp.2115-2126. July 2008.

[54] S. Bolognani, L. Tubiana, M. Zigliotto, "Extended Kalman Filter Tuning in Sensorless PMSM Drives", IEEE Trans. on Ind. Electron., vol. 39, no. 6, pp. 276 - 281, November 2003.

[55] A. Akrad, M. Hilairet, D. Diallo, "A Sensorless PMSM drive using a two stage Extended Kalman Estimator", In Proc. IECON'2008 Conf., pp. 2776-2781, Orlando, Florida, USA.

[56] B. Nahid-Mobarakeh, F. Meibody-Tabar, and F. Sargos "Mechanical Sensorless Control of PMSM With Online Estimation of Stator Resistance” IEEE Trans. On Ind. Applications, vol. 40 no. 2, pp. 457 - 471, March/April 2004

[57] Bendjedia, M.; Ait-Amirat, Y.; Walther, B.; Berthon, A "Sensorless control of hybrid stepper motor", in Proc. EPE Conf. 2007, pp. 1-10, CD-ROM.

[58] P. Brandstetter, M. Kuchar, D. Vinklarek: "Estimation Techniques for Sensorless Speed Control of Induction Motor Drive", in Proc. IEEE ISIE Conf., 2006, CD-ROM.

[59] A. Maalouf, L. Idkhajine, S. Le Ballois, E. Monmasson, "FPGAbased Sensorless Control of Brushless Synchronous Starter Generator for Aircraft Application", IET Electric Power Applications Journal, Accepted for publication in 2011.

[60] L. Idkhajine, E. Monmasson, A. Maalouf: "Fully FPGA-based Sensorless Control for AC Drive using an Extended Kalman Filter", In Proc. IECON'2009 Conf., pp. 2925-2930, Porto, Portugal.

[61] L. Idkhajine, E. Monmasson, A. Maalouf: "Extended Kalman Filter for AC Drive Sensorless Speed Controller - FPGA-Based solution or DSP-Based solution”, In Proc. ISIE'2010 Conf., Bari Italy.

[62] L. Idkhajine, E. Monmasson: "Optimized FPGA-based Extended Kalman Filter Application to an AC Drive Sensorless Speed Controller", In Proc. SPEEDAM'2010 Conf., pp. 1012-1017 Pisa Italy.

[63] Texas Instrument technical documents. Available in www.ti.com

[64] F. Zhengwei, J. E. Carletta, and R. J. Veillette, "A methodology for FPGA-based control implementation," IEEE Trans. Control Syst. Technol., vol. 13, no. 6, pp. 977-987, Nov. 2005.

[65] S. N. Murthy, W. Alvis, R. Shirodkar, K. Valavanis, W. Moreno, "Methodology for implementation of unmanned vehicle control on FPGA using system generator", In Proceedings ICCDCS'2004 Conference, CD-ROM.

[66] Oscar López, Jacobo Alvarez, Jesús Doval-Gandoy, et al., "Comparison of the FPGA implementation of two multilevel space vector PWM algorithms" IEEE Transactions on Industrial Electronics, vol.55, no. 4, pp. 1537-1547, April 2008.

[67] Da Zhang, Hui Li, "A Stochastic-Based FPGA Controller for an
Induction Motor Drive With Integrated Neural Network Algorithms," IEEE Trans. on Ind. Electronics, vol. 55, no. 2, pp. 551-561, Feb. 2008

[68] B. K. Bose, "Neural Network Applications in Power Electronics and Motor Drives - An Introduction and Perspective", IEEE Transactions on Ind. Electronics, vol. 54, no. 1, pp. 14-33, Feb. 2007

[69] X. Shao, D. Sun, "Development of a New Robot Controller Architecture with FPGA Based IC Design for Improved HighSpeed Performance", IEEE Trans. Ind. Informatics, vol. 3, no. 4, pp 312-321, 2007.

[70] Pedro Martín, Emilio Bueno et al., "A Methodology for Optimizing the FPGA Implementation of Industrial Control Systems", IECON '09. pp. 2831-2836, 2009.

[71] E. J. Bueno, Á. Hernández, et al., "A DSP and FPGA-based industrial control with high speed communication interfaces for grid converters applied to distributed power generation systems", IEEE Trans. on Industrial Electronics, vol. 56, no. 3, pp. 654-6691. March 2009.

[72] A. Nelson, T. Marcelo, "Custom Architectures for Fuzzy and Neural Networks Controllers," JCS\&T, vol. 2, no. 7, pp. 9-15, Oct. 2002.

[73] NEuroNet Roadmap. Future Prospects for Neural Networks, 2001 http://www.eunite.org/eunite/roadmap/02Roadmap1.pdf

[74] Special Section: "Neural Networks for Robotics", IEEE Trans. on Ind. Electronics, vol. 44, no. 6, Dec. 1997.

[75] Special Section: "Fusion of Neural Nets, Fuzzy Systems and Genetic Algorithms in Industrial Applications", IEEE Trans. on Ind. Electronics, vol.46, no.6, 1999.

[76] A. Gomperts, A. Ukil, F. Zurfluh, "Development and Implementation of Parameterized FPGA-Based General Purpose Neural Networks for Online Applications", IEEE Transactions on Industrial Informatics, Vol. 7, Issue 1, pp. 78-89, Feb. 2011.

[77] Q. N. Le, J.W. Jeon, "Neural-Network-Based Low-SpeedDamping Controller for Stepper Motor with an FPGA", IEEE Transactions on Industrial Electronics, Vol. 57, Issue 9, pp. 3167 3180, September 2010

[78] Ying-Shieh Kung, Ming-Shyan Wang, Tzu-Yao Chuang, "FPGAbased self-tuning PID controller using RBF neural network and its application in X-Y table", IEEE International Symposium on Industrial Electronics, 2009. ISIE 2009, pp. 694 - 699, July 2009.

[79] S. Sanchez-Solano, R. Senhadji, A. Cabrera, I. Baturone, C. J. Jimenez, A. Barriga, "Prototyping of Fuzzy Logic-Based Controllers Using Standard FPGA Development Boards," In Proc. 13th IEEE International Workshop on Rapid System Prototyping (RSP'02), 2002, CD-ROM.

[80] S.Poorani, T.V.S. Urmila Priya, K. Udaya Kumar, S. Renganarayanan: "FPGA Based Fuzzy Logic Controller For Electric Vehicle", Journal of the Institution of Electrical Engineers, Singapore, vol. 45, no. 5, 2005.

[81] D. Kim: "An implementation of fuzzy logic controller on the reconfigurable FPGA system," IEEE Trans. on Industrial Electronics, Vol. 47, no. 3, pp.703-715, Jun 2000.

[82] E. Lago, M. A. Hinojosa, C. J. Jiménez, A. Barriga, S. SánchezSolano, "Implementation of Fuzzy Controllers", 1997, http://citeseer.ist.psu.edu/46515.html

[83] F.J. Lin, D.H. Wang, P.K. Huang, "FPGA-based fuzzy slidingmode control for a linear induction motor drive," IEE Proceedings, Electric Power Applications, Vol. 152, no. 5, pp. 1137-1148, Sept 2005

[84] C. Cecati, F. Ciancetta, P. Siano, "A FPGA/fuzzy logic - Based multilevel inverter", IEEE International Symposium on Industrial Electronics, ISIE 2009, pp. 706-711. July 2009.

[85] Juang Chia-Feng, Lu Chun-Ming, Lo Chiang, and Wang Chi-Yen, "Ant Colony Optimization Algorithm for Fuzzy Controller Design and Its FPGA Implementation", IEEE Transactions on Industrial Electronics, vol. 55, no. 3, pp. 1453-1462, March 2008.

[86] S. Sanchez-Solano, E. del Toro, M. Brox, I. Baturone, A. Barriga "A design environment for synthesis of embedded fuzzy controllers on FPGAs", IEEE International Conference on Fuzzy Systems (FUZZ), pp. 1-8, July 2010

[87] M. Trimborne, "Optimizing Intelligent DAQ Devices with NI LabVIEW 8", National Instruments News, 21 Feb. 2006.

[88] J. Truchard, "Bringing FPGA design to application domain experts", 2010 International Conference on Field-Programmable Technology (FPT), pp iii, August 2010.

[89] T.N. Guoqiang Wang Tran, H.A. Andrade, "A graphical programming and design environment for FPGA-based hardware", 2010 International Conference on Field-Programmable Technology (FPT), pp. 337 - 340, August 2010. 
[90] P. Chandrashekhar Samuel, R.N. Gupta, "Wind energy conversion based on seven-level cascaded H-bridge inverter using LabVIEW FPGA", 2010 International Conference on Power, Control and Embedded Systems (ICPCES), pp. 1-6, December 2010.

[91] S.R. Verma Gadgil, M.S. D. Panse, K. Tuckley, "Sea State Monitoring HF Radar Controller Using Reconfigurable LabView FPGA", International Conference on Advances in Computing, Control \& Telecommunication Technologies, 2009. ACT'09. pp 395 - 397, December 2009.

[92] B. McMickell, P.J. Tanzillo, T. Kreider, K. Ilic, "Rapid development of space applications with responsive digital electronics board and LabVIEW FPGA", 2010 NASA/ESA Conference on Adaptive Hardware and Systems (AHS), pp. 78-81, June 2010.

[93] M. Kaminski, T. Orlowska-Kowalska, "FPGA realization of the neural speed estimator for the drive system with elastic coupling", 35th Annual Conference of IEEE Industrial Electronics, 2009. IECON '09, pp. 2831-2836. November 2009.

[94] C. Ortega, A.M. Tyrell: "A Hardware Implementation of an Embryonic Architecture Using Virtex FPGAs", Proceedings of the Third International Conference on Evolvable Systems: From Biology to Hardware, pp. 155 - 164, 2000, ISBN: 3-540-67338-5.

[95] W. Adi, K. Benkrid, "Adaptive and evolvable hardware security architectures", 2010 NASA/ESA Conference on Adaptive Hardware and Systems (AHS), pp. 194-198, June 2010.

[96] K. Glette, J. Torresen, M. Hovin, "Intermediate Level FPGA Reconfiguration for an Online EHW Pattern Recognition System", 2009 NASA/ESA Conference on Adaptive Hardware and Systems, AHS 2009, pp. 19-26, July 2009.

[97] P. Fernando, S. Katkoori, D. Keymeulen, R. Zebulum, A. Stoica, "Customizable FPGA IP Core Implementation of a GeneralPurpose Genetic Algorithm Engine", IEEE Trans. on Evolutionary Computation, vol. 14 , Issue 1, pp. 133-149, Feb. 2010.

[98] M. Samie, G. Dragffy, T. Pipe, "Bio-inspired self-test for evolvable fault tolerant hardware systems", NASA/ESA Conference on Adaptive Hardware and Systems (AHS), pp. 325-332, June 2010.

[99] P. Conmy, I. Bate, "Component-Based Safety Analysis of FPGAs", IEEE Trans. Ind. Informatics, vol. 6, no. 2, pp 195-205, 2010.

[100] B. M. Wilamowski, H. Yu, "Improved computation for Levenberg-Marquardt training”, IEEE Trans Neural Netw., 21(6), pp 930-937, 2010.

[101] B. M. Wilamowski, N. J. Cotton, O. Kaynak, G. Dundar, "Computing Gradient Vector and Jacobian Matrix in Arbitrarily Connected Neural Networks," IEEE Trans. on Ind. Electron., vol. 55, no. 10, pp. 3784-3790, Oct. 2008.

[102] A. Dinu, M. N. Cirstea: "A Digital Neural Network FPGA Direct Hardware Implementation Algorithm", Proc. of ISIE 2007, Vigo, Spain, pp.2307-2312

[103] A. Dinu, M.N. Cirstea, S.E. Cirstea: "Direct Neural Networks Hardware Implementation Algorithm", IEEE Trans. on Ind. Electronics, vol. 57, no. 5, pp.1845-1848, May 2010.

[104] A. Dinu, "FPGA Neural Controller for Three Phase Sensorless Induction Motor Drive Systems", PhD Thesis, De Montfort University, 2000.

[105] A. Aounis, M. McCormick, M.N. Cirstea: "A Novel Approach to Induction Motor Controller Design and Implementation", Proc. of IEEE Power Conversion Conference (PCC), Osaka, pp.993-998, April 2002.

[106] A. Tisan, et al, "Architecture and Algorithms for Synthesizable Neural Networks with On-Chip Learning", 8-th International Symposium on Signals, Circuits and Systems. ISSCS 2007, (1), pp. $265-268,2007$.

[107] A. Tisan, M.N. Cirstea, S. Oniga, A. Buchman, "Artificial Olfaction System with Hardware On-chip Learning Neural Networks", Proc. of IEEE Int. Conference on Optimisation of Electrical and Electronic Equipment (OPTIM 2010), Brasov, Romania, 20-22 May 2010, vol., pp. 884-889.

[108] D. Zhang, H. Li, S.Y. Foo: “A Simplified FPGA Implementation of Neural Network Algorithms Integrated with Stochastic Theory for Power Electronics Applications", Industrial Electronics Conference, IECON'05, Raleigh, USA, pp.1018-1024, 2005.

[109] H. Amin, et all: "Piecewise linear approximation applied to nonlinear function of a neural network", IEE Proc. Circuits, Devices Sys., 1997, 144, (6), pp. 313-317.

[110] M.T. Tommiska: "Efficient digital implementation of the sigmoid function for reprogrammable logic", IEE Proceedings Computers and Digital Techniques, number 6, pp. 403-411, 2003.

[111] O. Cheng, W. Abdulla, Z. Salcic, "HardwareSoftware Codesign of Automatic Speech Recognition System for
Embedded Real-Time Applications", IEEE Trans. on Ind. Electron., vol. 58, no. 3, pp. 850-859, March 2011.

[112] L. Siew-Kei, T. Srikanthan, C.T. Clarke, "Selecting Profitable Custom Instructions for Area-Time-Efficient Realization on Reconfigurable Architectures", IEEE Trans. on Ind. Electron., vol. 58, no. 3, pp. 850-859, Oct. 2009. 